\title{
Tungstate Sorption Mechanisms on Boehmite: Systematic Uptake Studies and X-ray Absorption Spectroscopy Analysis
}

\author{
HyuckHur $^{\mathrm{a}, \dagger}$ and Richard J. Reeder ${ }^{\mathrm{b}, *}$ \\ aa Department of Chemistry, Stony Brook University, Stony Brook, NY 11794 USA \\ ${ }^{\mathrm{b}}$ Department of Geosciences, Stony Brook University, Stony Brook, NY 11794USA
}

Revised: 31 August 2015

\begin{abstract}
*Corresponding author, Address:Department of Geosciences, Stony Brook University, Stony Brook, NY 11794-2100USA. Phone: +1-631-632-8208; Email: rjreeder@ stonybrook.edu

${ }^{\dagger}$ Current address:Department of Earth and Environmental Sciences, Korea University, Seoul, Korea
\end{abstract}

1

(C) 2015. This manuscript version is made available under the Elsevier user license http://www.elsevier.com/open-access/userlicense/1.0/ 


\section{Graphical Abstract}

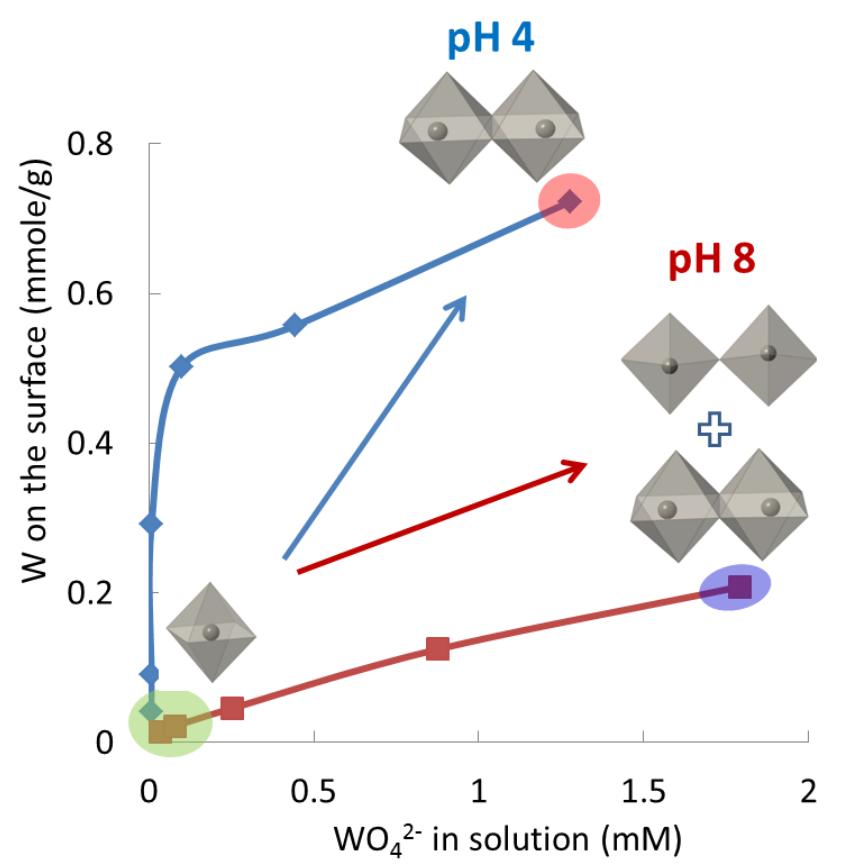

47

48

49

50

51

52

53

54

55

56

57

58

59

60

61

62

63

64

65 


\begin{abstract}
Mechanisms of tungstate sorption on the mineral boehmite $(\gamma-\mathrm{AlOOH})$ were studied using batch uptake experiments and X-ray absorption spectroscopy. Batch uptake experiments over the $\mathrm{pH}$ range 4-8 and $[\mathrm{W}]=50-2000 \mu \mathrm{M}$ show typical oxyanion behavior, and isotherm experiments reveal continued uptake with increasing tungstate concentration without any clear uptake maximum. Desorption experiments showed that sorption is irreversible at $\mathrm{pH} 4$ and partly reversible at $\mathrm{pH}$ 8. Tungsten $\mathrm{L}_{1^{-}}$and $\mathrm{L}_{3}$-edge XANES spectroscopy indicatesthat all sorbedtungstates are octahedrally coordinated, even though the dominant solution species at $\mathrm{pH} 8$ is a tetrahedral monotungstate.Tungsten $\mathrm{L}_{3}$-edge EXAFS analysis shows that sorbedtungstate occurs as polymeric form(s), as indicated by the presence of cornerand edge-sharing of distorted tungstate octahedra. The occurrence of polymeric tungstate on the surface at $\mathrm{pH} 8$ indicates that sorption is accompanied by polymerization and a coordination change from tetrahedral (in solution) to distorted octahedral (on the surface). The strong tendency for tungstate polymerization on boehmitecanexplain the continued uptake without an apparent maximum in sorption, and the limited desorption behavior. Our results provide the basis for a predictive model of tungstate uptake by boehmite, which can be important for understanding tungstate mobility, toxicity, and bioavailability.
\end{abstract}

Keywords: tungstate, polytungstate, isopolytungstate, tungsten, boehmite, $\mathrm{AlOOH}$, sorption, polymerization, X-ray absorption spectroscopy

\title{
1. Introduction
}

Until recently, little consideration has been given to the environmental impact of tungsten. This industrially important metal has been widely thought to be nontoxic in its pure or alloyed forms [1, 2]. Oxidation of metallic forms of tungsten results in dissolution and formation of soluble anions that are mobile in aquatic and soil systemsunder appropriate conditions of $\mathrm{pH}$ and redox potential [3].The most 
stable oxidation state in surface environments is $\mathrm{W}(\mathrm{VI})$, tungstate, which forms many different oxyanions, including monooxyanion and polyoxyanion forms [4-6]. The study of tungstate behavior in the environment is complex due to the occurrence of multiple species associated with polymerization and their interactions with environmental materials.It is now recognized that the presence of dissolved tungstate may lead to adverse environmental effects, including soil acidification as well as toxic effects on plants, soil microorganisms and invertebrates [7-9]. Recent studies have shown that the toxicity of tungstate is related to its speciation. Strigul et al. studied toxicities of tungstate species in fish, and reported that polymeric tungstates were more toxic than monotungstate [10]. Investigation of tungstate behavior in aqueous systems, including its toxicity, has become increasingly important as industrial applicationsand releases to the environment have escalated.

Tungstate oxyanion speciationin solution depends on $\mathrm{pH}$ as well as total $\mathrm{W}$ concentration, showing some similarities with molybdenum oxyanionbehavior $[11,12]$. Like molybdate, the dominant oxyanion species of tungstate at basic $\mathrm{pH}$ is monomeric $\mathrm{WO}_{4}{ }^{2-}$ with tetrahedral coordination. $\mathrm{As} \mathrm{pH}$ is decreased, tungstate forms polymeric species havingmainly octahedral coordination as shown for selected species in Figure 1 and described further in Supporting Information. Polymerization is favored with increasing tungstate concentration. In the VIB group,the tendency for polymerization increases with increasing atomic number, so that occurrence of polymeric forms of tungstate may be more common than molybdate for equivalent conditions [11].Tungstate speciation has been studied by several researchers with ${ }^{187} \mathrm{~W}$ nuclear magnetic resonance (NMR) spectroscopy, electrospray ionization mass spectrometry (ESI-MS), and Raman spectroscopy[4, 5, 13, 14].These studies have shown that general trends of tungsten polymerization are known under acidic conditions, yet the mechanismsare still not completely understood.Furthermore, the kinetics of formation of some polymeric species is sluggish, so that equilibrium speciation is not necessarily obtained over the time scale of laboratory experiments $[4,5,11]$.

Sorption processes on mineral surfaces play an important role in regulating the distribution and mobility of trace metals in natural aquatic and soil systems.Tungstate has been shown to adsorb strongly 
on iron oxyhydroxide mineral surfaces at low $\mathrm{pH}$ conditions[15-17].Hernandez performed sorption experiments and IR spectroscopy to characterize $\mathrm{H}_{2} \mathrm{~W}_{12} \mathrm{O}_{40}{ }^{6-}$ sorption on iron and aluminum (hydr)oxide sufaces [15]. Gustafsson used a 2-pK Diffuse Layer Model and a1-pKCD-MUSIC model to describe tungstate (and molybdate) sorption on ferrihydrite, accounting for monomeric and polymeric tungstates to fit the experimental data for both models[17]. The competitive sorption of tungstate and other oxyanions on goethite was studied by $\mathrm{Xu}$ et al.[16]. Tungstatesorption was found to be strongly competitive with molybdate and phosphate at the surface, whereas silicate and sulfate sorption were affected minimally by tungstate. Tungstate speciation in natural soils was studied by Clausen and Korte [2] and Bednar et al. [3], who found tungstate forming polymeric specieswith phosphate and silicate, and proposed a general transformation pathway fortungstates in nature.However, little is known of the influence that tungstate speciation plays in sorption behavior over a broader $\mathrm{pH}$ range and on other mineral sorbents. This fundamental information is important inasmuch as it may control tungstate mobility, toxicity, and bioavailability in natural systems.

In the present study, we investigate tungstate sorption behavior on the aluminum oxyhydroxide mineral boehmite, $\gamma$-AlOOH,over the tungstate concentration range 5-2000 $\mu \mathrm{M}$. Although the lower end of this concentration range is more relevant environmentally, dissolved tungsten concentrations as high as $400 \mathrm{mg} / \mathrm{L}(2175 \mu \mathrm{M})$ have been reported at highly contaminated sites [2]. Boehmite occurs naturally as a common weathering product and is an effective sorbent for both cations and anions[18-21].Tungsten $\mathrm{L}_{1^{-}}$ and $\mathrm{L}_{3}$-edge XANES spectroscopy was used to distinguish coordination environments around $\mathrm{W}$ atoms. The local structure and coordination of tungsten at the surface were determined using tungsten $\mathrm{L}_{3}$-edge EXAFS, which allowed us to further characterize the binding mechanism as well as the dependence of tungstate sorption on environmental parameters such as $\mathrm{pH}$, metal concentration, and ionic strength. This initial work provides a foundation for subsequent studies of tungstate sorption on other solids. The findings also have possible implications for tungsten toxicity in natural environments.

\section{Materials and Methods}




\subsection{Sorbent}

Boehmite from CONDEA Chemie GmbH was used in this study. Powder XRD was used to confirm the structure, and no other phases were detected. The specific surface area of the boehmitewas found to be $136 \mathrm{~m}^{2} / \mathrm{g}$ by five-point $\mathrm{N}_{2}$ Brunauer-Emmett-Teller (BET) analysis. The point of zero charge (PZC) determined for this material in previous studies lies in the range 8.6-9.1[19, 22].

\subsection{Batch uptake experiments}

Batch uptake experiments were conductedover a range of tungsten concentrations, from 50 to $2000 \mu \mathrm{M}$, at $\mathrm{pH} 4,6.5$, and 8. Ionic strength of 0.01 or $0.1 \mathrm{M}$ was achieved using $\mathrm{NaCl}$ as a background electrolyte. Based on tungstate speciation calculations (see Supporting Information), $\mathrm{pH} 4$ and 8 represent solution conditions for which polytungstates and monotungstate,respectively, representthe major components in the solutionover the studied concentration range (Figure 1).The pH 6.5 condition represents a mixture of monomeric and polymeric species, with proportions varying depending on total $\mathrm{W}$ concentration. The boehmite suspensions were equilibrated overnight before being titrated to the desired $\mathrm{pH}$ using $\mathrm{HCl}$ or $\mathrm{NaOH}$. After an additional 24 h equilibration time, a pre-determined amount of a0.1 M or $0.01 \mathrm{M} \mathrm{Na}_{2} \mathrm{WO}_{4}$ stock solution was added to $1 \mathrm{~g} / \mathrm{L}$ boehmite suspensions to achieve the target $\mathrm{W}$ concentration. Small amounts of $0.1 \mathrm{M} \mathrm{HCl}$ or $\mathrm{NaOH}$ were used to adjust the $\mathrm{pH}$ after adding the $\mathrm{Na}_{2} \mathrm{WO}_{4}$ solution to the suspension. After 24 hours of equilibration on a shaker table, the suspensions were centrifuged for $10 \mathrm{~min}$ at 10,000 rpm, and $10 \mathrm{~mL}$ aliquots of supernatant werecollected from each sample. Tungsten concentration in the aliquots was measured with direct coupled plasma atomic emission spectroscopy (DCP-AES) to calculate the amount of tungsten sorbed on the boehmite surface. Selected samples were filtered using a vacuum pump, and the wet pastes were prepared for XAS analysis.

Tungstate stock solutions were prepared using $\mathrm{Na}_{2} \mathrm{WO}_{4} \cdot 2 \mathrm{H}_{2} \mathrm{O}$ (Alfa Aesar) and deionized water. The stock solutions were pre-titrated to target $\mathrm{pH}$ values prior to addition to the suspensions. To determine if pre-titration influenced sorption results, a set of parallel experiments was performed using freshly 
prepared stock solution without pre-titration. No discernible differences were found between results for these different stock solutions in terms of uptake or XAS.

All sorption experiments were performed under atmospheric conditionsand no effort was made to purge $\mathrm{CO}_{2}$ from the solutions. However, containers holding suspensions were kept sealed except for addition of stock solution and adjustments to $\mathrm{pH}$, so $\mathrm{CO}_{2}$ exchange was restricted.Nevertheless, the presence of dissolved $\mathrm{CO}_{2}$ is expected to have some influence on the surface charge of boehmite in suspensions as a result of sorption.Su and Suarez examined the effect of carbonate sorption on surface charge of gibbsite $\left(\mathrm{Al}(\mathrm{OH})_{3}\right)$ and amorphous aluminum hydroxide, and showed that the observed PZC decreased by 0.5-0.7 $\mathrm{pH}$ units in suspension titrated with sodium carbonate solution [23].

\subsection{Desorption experiments}

Desorption experiments were conducted following the conclusion of sorption reactions (as described above) at two concentrations: 200 and $1000 \mu \mathrm{M}$. The final suspensions after $24 \mathrm{~h}$ reaction were centrifuged for $10 \mathrm{~min}$ at $10,000 \mathrm{rpm}$, the solution was discarded, and the moist particles were resuspended in tungstate-free solution with the same $\mathrm{pH}$ condition and background electrolyte concentration. Aliquots $(5 \mathrm{~mL})$ were taken at designated time periods, and $\mathrm{W}$ concentration in the solution was measured by DCP-AES to allow calculation of sorbed W.

\subsection{X-ray absorption spectroscopy}

\subsubsection{Tungsten $\mathrm{L}_{1^{-}}$and $\mathrm{L}_{3}$-edge EXAFS and XANES}

Tungsten $\mathrm{L}_{1^{-}}$and $\mathrm{L}_{3}$-edge XANES and $\mathrm{L}_{3}$-edge EXAFS data were collected at beamlines $20 \mathrm{BM}$ and 12BM at the Advanced Photon Source (APS, Argonne National Laboratory) and at X11A and X19A at the National Synchrotron Light Source (NSLS, Brookhaven National Laboratory). Spectra were collected at the $\mathrm{W} \mathrm{L}_{1}$ and $\mathrm{L}_{3}$ edges using $\mathrm{Si}(111)$ monochrometer crystals with detuning of 10-30\%. Energy calibration wasperformed with a Ga filter (K-edge, $10.367 \mathrm{keV}$ ) or a W metal foil for the $\mathrm{W} \mathrm{L}_{3^{-}}$ 


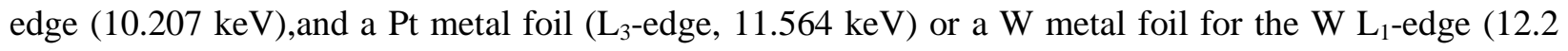
$\mathrm{keV}$ ). The monochrometer was calibrated by assigning the indicated energy to the first peak of the derivative of the edge spectrum of the element used for calibration.

EXAFS and XANES spectra for model compounds were collected in transmission mode. Model compounds were mixed with boron nitride to achieve the proper edge step, and then loaded into Lucite sample holders and sealed using two layers of Kapton tape. All spectra for sorption samples and solutions were taken in fluorescence mode using a partially implanted planar silicon (PIPS) detector at the NSLS and a 13-element Ge detector at the APS. Wet pastesobtained from vacuum filtration of reacted suspensions were sealed in Lucite sample holders with Kapton tape and stored in sealed polyethylene bags with wet tissues to prevent drying. Samples were placed at a $45^{\circ}$ angle to the incident beam for fluorescence measurements. Multiple spectra were routinely collected and averaged to achieve acceptable signal/noise.

\subsubsection{Tungsten $\mathrm{L}_{3}$-edge EXAFS fitting}

Data analysis was conducted using the programs iFeffit[24] and WinXAS[25]. Shell by shell fitting was performed in real space using phases and amplitudes calculated by FEFF7 [26] based on published structures of selected reference compounds. Fitting strategies were developed and validated using EXAFS data collected from model compounds representing the wide variety of $\mathrm{W}(\mathrm{VI})$ coordination environments, including isolated tetrahedra and octahedra and various polymeric forms. Structures of these tungstate model compounds and EXAFS fit results are described in Supporting Information.

\section{Results}

3.1Batch uptake trends

The effect of $\mathrm{pH}$ on tungstate sorption by boehmite was investigated at an initial tungstate concentration of $50 \mu \mathrm{M}$ at two ionic strength conditions using $\mathrm{NaCl}$ as a background electrolyte (Figure 

range 5.0-5.5, and decreasing sorption with increasing $\mathrm{pH}$, approaching minimum values near and above the PZC of boehmite, 8.6-9.1. A smaller decrease in sorption is observed at $\mathrm{pH}$ values below 5. The efficiency of $\mathrm{W}$ sorption is slightly affected by ionic strength.Slightly greater tungstate sorption is observed at $0.1 \mathrm{M} \mathrm{NaCl}$ for $\mathrm{pH}>6$, while the boehmitesuspensionat $0.01 \mathrm{M} \mathrm{NaCl}$ shows slightly greater sorption at $\mathrm{pH}<5$. The lower tungstate sorption with $0.1 \mathrm{M} \mathrm{NaCl}$ at $\mathrm{pH}<5$ might beexplained by complexation with $\mathrm{Na}^{+}$in solution. The polymeric tungstate with large negative charge, e.g., $\mathrm{H}_{2} \mathrm{~W}_{12} \mathrm{O}_{40}{ }^{6-}$, could form electrostatic bonds with $\mathrm{Na}^{+}$thereby reducingpolytungstatesorption on boehmite at higher $\mathrm{NaCl}$ concentrations. The greater tungstate sorption at $0.1 \mathrm{M} \mathrm{NaCl}$ above $\mathrm{pH} 5$ is similar to observations by Li et al.[27], whostudied phosphate sorption onboehmite from the same source (CONDEA Chemie $\mathrm{GmbH}$ ). These authors attributed greater phosphate uptake at higher $\mathrm{NaCl}$ concentration to $\mathrm{Na}^{+}$coadsorption on the surface. However, it is possible that the greater sorption observed in $0.1 \mathrm{M} \mathrm{NaCl}$ suspensions at $\mathrm{pH}>7$ may reflect decreased competition with sorbed carbonate, which is expected to be less at the higher ionic strength $[28,29]$. At the lower ionic strength $(0.01 \mathrm{M})$, greater carbonate sorption would be more effective in shifting the PZC to lower values, so that less tungstate uptake would be expected.

Uptake experiments were performed to construct isotherms in the range 50 to $2000 \mu \mathrm{M}$ total tungstate at $\mathrm{pH} \mathrm{4,6}$ and 8 (Figure 3). These $\mathrm{pH}$ conditions were chosen to represent solutions in which polytungstate species $(\mathrm{pH} 4)$ or a monotungstate $(\mathrm{pH} 8)$ dominates, or in which a mixture is present $(\mathrm{pH}$ 6), as indicated by the speciation calculations described above. We performed initial kinetic experiments of tungstate sorption (initial $\left[\mathrm{WO}_{4}{ }^{2-}\right]=200 \mu \mathrm{M}$, and $0.19 \mathrm{mmol} / \mathrm{g}\left(0.84 \mathrm{~W} / \mathrm{nm}^{2}\right.$ ) sorbedon boehmiteat $\mathrm{pH}$ 4), which confirmed that sorption is fast, with $\sim 80 \%$ of the equilibrium amount of tungstate sorbed within the initial few hours, and $>90 \%$ sorbed within $24 \mathrm{~h}$. On this basis, all subsequent sorption experiments were conducted over $24 \mathrm{~h}$ duration. In all $\mathrm{pH}$ conditions, the amount of $\mathrm{W}$ sorbed on the surface increased as $\mathrm{W}$ concentration in the solution increased, without reaching a maximum in the concentration range 
studied. As we expected from the $\mathrm{pH}$ edge experiments, W showed greatest sorption at $\mathrm{pH}$ 4. The tungstate surface coverage increased from $0.04 \mathrm{mmol} / \mathrm{g}$ to $0.72 \mathrm{mmol} / \mathrm{g}\left(0.18\right.$ to $\left.3.19 \mathrm{~W} / \mathrm{nm}^{2}\right)$ at $\mathrm{pH} 4$, and increased from 0.01 to $0.21 \mathrm{mmol} / \mathrm{g}\left(0.04\right.$ to $\left.0.93 \mathrm{~W} / \mathrm{nm}^{2}\right)$ at $\mathrm{pH} 8$.

\subsection{Desorption Experiments}

Desorption experiments were performed at 200 and $1000 \mu \mathrm{M}$ initial $\mathrm{W}$ concentrations at $\mathrm{pH} 4$ and 8 to establish sorption reversibility. Tungstate sorbed for $24 \mathrm{~h}$ on boehmite at $\mathrm{pH} 4$ showed largely irreversible behavior in desorption experiments, with more than $95 \%$ of the tungstate remaining on the surface for both initial concentrations studied. Sorption was found to be partly reversible at $\mathrm{pH} 8$, as shown in Figure 4. Approximately $25-30 \%$ of the tungstate on the surface was released into the solution at $\mathrm{pH} 8$, with slightly greater release at $200 \mu \mathrm{M}$ than at $1000 \mu \mathrm{M}$. These desorption results imply different sorption mechanisms on the boehmite surface at $\mathrm{pH} 4$ and $\mathrm{pH} 8$. X-ray absorption spectroscopy was used to provide further insight into dominant sorption mechanisms.

\subsection{Tungsten $\mathrm{L}_{1}$ - and $\mathrm{L}_{3}$-edge XANES}

\subsection{1 $\mathrm{W} \mathrm{L} \mathrm{L}_{1}$-edgeXANES}

Both the $\mathrm{L}_{1^{-}}$and $\mathrm{L}_{3}$-edge XANES spectraof tungsten have proven useful for characterizing tungstate coordination environments[30].A pre-edge feature is observed in the $\mathrm{L}_{1}$ edge, associated with electron transitions from $2 \mathrm{~s}$ to unoccupied valence orbitals. This transition is dipole forbidden in symmetric octahedral environments, but allowed for tetrahedral and distorted octahedral environments. Previous studies have shown that the intensity and shape of the pre-edge feature relate to coordination and degree of (octahedral) distortion[31]. Reference compounds that contain only tetrahedrally coordinated W(VI), which include $\mathrm{Na}_{2} \mathrm{WO}_{4} \cdot 2 \mathrm{H}_{2} \mathrm{O}$ and $\mathrm{CaWO}_{4}$, exhibit a prominent high-intensity peak in the preedge region (Figure S3 in Supporting Information).In contrast, reference compounds with only octahedral coordination, such as $\mathrm{Ba}_{2} \mathrm{NiWO}_{6}, \mathrm{TBA}-\mathrm{W}_{6} \mathrm{O}_{19}$, phosphotungstic acid, and $\mathrm{WO}_{3}$, display pre-edge peaks 
that are lower in intensity anddifferent in shape than tetrahedral compounds. Furthermore, because the intensity of the pre-edge feature for octahedrally coordinated compounds is sensitive to the degree of distortion, differences in pre-edge intensity and shape are evident among the various octahedralcompounds (see section 4 in Supporting Information for further description). The pre-edge features for all the sorption samples are found to be similar to one another, with pre-edge intensities lower than those in tetrahedral reference compounds, and most closely matching reference samples with distorted octahedral coordination, such asphosphotungstic acid.

\subsection{2 $\mathrm{W} \mathrm{L}_{3}$-edge XANES}

Tungsten $\mathrm{L}_{3}$-edge XANES has been studied less than the $\mathrm{L}_{1}$-edge. Recently, Yamazoe et al. demonstrated that second derivatives of the $\mathrm{L}_{3}$-edge show differences corresponding to coordination environment, analogous to the ligand field splitting of $5 \mathrm{~d}$ orbitals observed for different coordination environments of $\mathrm{Mo}(\mathrm{VI})$ [30].Second derivative $\mathrm{L}_{3}$-edge XANES spectra of reference samples containing only W(VI) tetrahedra show a single minimum with a weak shoulder on the low-energy side, as observed for $\mathrm{Na}_{2} \mathrm{WO}_{4} \cdot 2 \mathrm{H}_{2} \mathrm{O}$ and $\mathrm{CaWO}_{4}$ (Figure5). Model compounds containing only W(VI) octahedra show splitting in the second derivatives to create two minima. Yamazoe showed that the shapeof the minima and the degree of splittingbetween them are dependent on the coordination type and the degree of distortion of the octahedral [30]. Figure 5also shows second derivatives of $\mathrm{W} \mathrm{L}_{3}$-edge XANES spectra of the lowest $\mathrm{W}$ concentration sorption samples $(5 \mu \mathrm{M})$ at $\mathrm{pH} 4$ and 8 , compared with tetrahedral and octahedral model compounds. Findings for higher concentration sorption samples are summarized in Supporting Information (Table S2). Second derivative spectra of both sorption samples display two well separated minima, consistent with distorted octahedral coordination of W(VI). Notably, these second derivative spectra are nearly identical for samples at both $\mathrm{pH}$ conditions. In Figure 5, sorption samples are clearly distinguished from $\mathrm{Na}_{2} \mathrm{~W}_{2} \mathrm{O}_{7}$, which contains both tetrahedral and octahedral W(VI). Although it 

component in the sorption samples is clearly octahedral, and the octahedraare distorted on average.

\subsubsection{Combined $\mathrm{L}_{1}$-edge and $\mathrm{L}_{3}$-edge XANES}

By combining the pre-edge peak area from $\mathrm{L}_{1}$-edge XANES with the energy separationbetween minima in the $\mathrm{L}_{3}$-edge second derivatives, clear differences are evident among $\mathrm{W}(\mathrm{VI})$ coordination environments (Figure 6). $\mathrm{Na}_{2} \mathrm{WO}_{4} \cdot 2 \mathrm{H}_{2} \mathrm{O}$ and $\mathrm{CaWO}_{4}$, both with tetrahedrally coordinated $\mathrm{W}$, lie at one end of a trend, distinguished by a large pre-edge area in $\mathrm{W} \mathrm{L}_{1}$-edge XANES and a small energy separation in $\mathrm{W} \mathrm{L}_{3}$-edge second derivatives. On the other end of this trend lies $\mathrm{Ba}_{2} \mathrm{NiWO}_{6}$, with perfect octahedral coordination, havinga relatively small pre-edge area and a large energy seperation in the second derivative minima. Reference compounds with more distorted octahedra, such as $\mathrm{WO}_{3}$, phosphotungstic acid, and sodium metatungstate, lie at intermediate positions along the trend, reflecting intermediate values of pre-edge peak area and energy separation.All of the sorption samples are tightly clustered at an intermediate position along the trend (within the yellow circle, Figure 6). Their proximity near $\mathrm{Na}_{2} \mathrm{~W}_{2} \mathrm{O}_{7}$, which contains both tetrahedral and octahedral $\mathrm{W}(\mathrm{VI})$, might suggest that the sorption samples also contain mixtures of $\mathrm{W}(\mathrm{VI})$ tetrahedra and octahedra. However, the second derivative spectrafor the $\mathrm{L}_{3^{-}}$ edge (Figure 5) clearly indicate that all sorption samples have octahedrally coordinated W(VI), as described in the previous section.

\subsection{W L $\mathrm{W}_{3}$-edge EXAFS}

\subsubsection{Model compounds and tungstate reference solutions}

$\mathrm{W} \mathrm{L}_{3}$-edge EXAFS provides further insight to local structure of sorbed W(VI). Fitting of selected reference compounds and aqueous tungstate solutions demonstrated that it is possible to distinguish polymeric and monomeric species, and allowed us to formulate a fitting strategy for sorption samples. EXAFS chi functions and corresponding Fourier transforms are shown in Figure 7 for selected model 

Information Table S3.

The $1 \mathrm{mM}$ pH4 and 8 solutions show distinct differences intheir chi curves and FT magnititudes (Figure 7).The chi function for the $\mathrm{pH} 8$ solution shows a single beat, and the FT magnitude shows a corresponding single peak, similar in character to the $\mathrm{Na}_{2} \mathrm{WO}_{4} \cdot 2 \mathrm{H}_{2} \mathrm{O}$ reference sample, which contains only tungstate tetrahedra. The $\mathrm{pH}$ 8solution data were best fit with a single shell of W-O atoms at $1.78 \AA$, which is characteristic of tetrahedral $\mathrm{W}(\mathrm{VI})$ coordination, and consistent with the dominance of monomeric tetrahedral species expected at $\mathrm{pH} 8$ and confirmed by electrospray ionization mass spectroscopy (ESI-MS) (see Supporting Information). In contrast, the $\mathrm{pH} 4$ solution shows several oscillations with different phases in the chi curve, with evident splitting in the first two oscillations, similar to the splitting observed for the polymeric reference samples (Figure 7). The FT magnitude for the pH 4 solution shows a split first peak with weaker peaks at higher R. The split first peak compares with the polymeric reference compounds but with a different asymmetry in intensities. The distinguishing feature of polytungstates is connected W(VI) octahedra, whichshould beevident in FT data as peaks at greater distances, like those seen in model compounds (Figure 7). The peaks in the range 3.3-3.8 $\AA$ are consistent with edge- and corner-sharing of tungstate octahedra, and confirmed by fitting reference compounds. At $\mathrm{pH} 4$, polymerization is favored, with $\mathrm{H}_{2} \mathrm{~W}_{12} \mathrm{O}_{40}{ }^{6}$ being the dominant aqueous species based on the calculations (Figure 1; see Supporting Information)and confirmed by the ESI-MS results(Figure S2 in Supporting Information) showing the likely presence of multiple polytungstate speciecs. The structure of $\mathrm{H}_{2} \mathrm{~W}_{12} \mathrm{O}_{40}{ }^{6-}$ contains two distinct $\mathrm{W}-\mathrm{W}$ distances, $\sim 3.3 \AA$ for edge-sharing and 3.8Åfor corner-sharing. These distances are consistent with our EXAFS result for the solution at pH 4. However, it is not possible to uniquely identify the metatungstate species or to rule out the presence of other polymeric tungstate species, inasmuch as they share the distinctive edge- and corner-sharing of W(VI) octahedra, and would not be distinguishable by EXAFS.Nevertheless, the EXAFS results confirm 
the dominance of polymeric tungstate species in the $\mathrm{pH} 4$ solution, whereas the $\mathrm{pH} 8$ solution contains monomeric tetrahedral tungstate.

\subsubsection{Tungstate sorption samples}

The $\mathrm{W} \mathrm{L}_{3}$-edge EXAFS of tungstate-sorbed boehmite samples at different tungstate concentrations and $\mathrm{pH}$ conditions are compared in Figure 8. Based on the experimental isotherms, we chose sorption samples for EXAFS study at three total W concentrations: 5, 200, and $1000 \mu \mathrm{M}$. At $\mathrm{pH} 4$, these concentrations result in coverages of $0.022,0.197$, and $0.557 \mathrm{mmol} / \mathrm{g}\left(\right.$ or $0.10,0.87$, and $\left.2.47 \mathrm{~W} / \mathrm{nm}^{2}\right)$. The surface coverages at $\mathrm{pH} 8$ are $0.007,0.014$, and $0.125 \mathrm{mmol} / \mathrm{g}\left(0.03,0.06\right.$, and $\left.0.55 \mathrm{~W} / \mathrm{nm}^{2}\right)$. The most striking observation is that the chi functions are broadly similar for all samples, yet small, distinguishing differences are evident (Figure 8). All chi functions show a split first oscillation and a sharp second oscillation apparently from interference with a second or additional beat. The200 and $1000 \mu \mathrm{MpH} 4$ samplesexhibit an asymmetryin the split first oscillation in the chi function compared to other samples, as well as a different or additional phase obvious at higher $\mathrm{k}$ values.

The corresponding FT magnitudes show generally similar first peaks, corresponding to first-shell W-O coordination, but important differences are evident in weaker peaks at higher $\mathrm{R}$ values. For the lowest concentration samples, $5 \mu \mathrm{M}$ tungstate, no discernible differences are evident between $\mathrm{pH} 4$ and 8 conditions. We note that FT magnitudes for the 200 and $1000 \mu \mathrm{M}$ pH 8 sorption samples are nearly identical to one another, exhibiting peaks in the range 3.0-3.5 (Figure 8, not corrected for phase shifts), which are consistent with backscattering from $\mathrm{W}$ or $\mathrm{Al}$. ThesepH 8 samples are distinct from the same concentration samples at $\mathrm{pH} 4$, which show different peaks in the range 3.0-3.5 $\mathrm{A}$. The $5 \mu \mathrm{M}$ samples are different from the higher concentration samples at both $\mathrm{pH} 4$ and $\mathrm{pH} 8$, as indicated by the absence of distinct peaks at 3.0-3.5 . Fitting described in the following section provides further insight about local structure.

3.5 $\mathrm{W} \mathrm{L}_{3}$-edge EXAFS fitting 
The primary challenges in fitting $\mathrm{W} \mathrm{L}_{3}$-edge EXAFS spectra for soprtion samples arise from the existence of multiple, overlapping paths for polymeric species and the likely existence of multiple species, particularly at high tungsten concentrations.A distinction between tetrahedral and octahedral coordination can be made on the basis of average, first-shell W-O distances. The average W-O distance in tetrahedral tungstates is $\sim 1.8 \AA$, whereas polytungstates have longer average W-O distances $(\sim 1.95 \AA)$, reflecting their octahedral coordination.However, the octahedral coordination in polytungstates is invariably distorted, resulting in a range of individual W-O distances (1.76-2.4 $\AA$ ). Because of the distortion observed in $\mathrm{W}$ octahedra, fitting typically required two or more $\mathrm{W}-\mathrm{O}$ paths, whereas tetrahedral W was best fit with a single W-O path. Although the common polymeric tungstate species differ in the details of their configuration, they share several common characteristics that distinguish them from monomeric, tetrahedral species. Tungstate octahedra may be connected by corner- and/or edge-sharing, and the corresponding W-Wdistances can generally be distinguished. Although some overlap exists for the range of characteristic distances for edge- vs. corner-sharing, peaks in the range 3.3-3.8 Athat are fit well by W-W paths provide clear evidence of polymeric tungstates, even if the exact species cannot be identified. Further complications in fitting may result if multiple polymeric species exist.

The general fitting procedure followed three steps. First, the main peak corresponding to first-shell W-O backscattering was fit using one or more paths. The best fits for sorption samples were typically achieved using two or three W-O paths for the first main peak in FT magnititude. Second, a near-linear OW-O MS path was introduced. Multiple scattering is found to have an effectmainly at low $\mathrm{k}$ in the chi functions, and accounting for it was found to be necessary to achieve acceptable agreementwith the observed splitting in the first oscillation near 3.5-5 $\AA^{-1}$. Finally, the peaks in the range 3.0-3.8 $\AA$ were fit with $\mathrm{W}-\mathrm{W}$ and/or W-Al paths. Reference compounds were fit to validate the strategy described, however, their coordination numbers were constrained to be consistent with known structure data.

The EXAFS data for the lowest concentration sorption samples were fit first with single scattering paths for octahedral coordination. We constrained total coordination number of the first shell to 6 if the fit 
results were not reasonable with floating coordination numbers. We assumed that a W-Al path contributesto the EXAFS signal for all sorption samples, so the parameters of a W-Al path, obtained from the fit results of the $5 \mu \mathrm{M}$ sorption sample, were fixed in the fitting process. The fit results of higher concentration samples were compared with and without a W-Al path adopted from the result of the lowest concentration sample. We also included up to two W-W paths, corner- and edge-sharing, to fit the second shell for the higher concentration smples.

Fit results for all sorption samples are summarized in Table 1, and fits for representative samples are shown in Figure 8. Both $5 \mu \mathrm{M}$ tungstate sorption samples ( $\mathrm{pH} 4$ and 8)were fit best with two W-O paths(1.75 and $2.14 \AA$ ) for the first peak with a combined coordination number $(\mathrm{CN})$ of $6.3( \pm 1.2)$. This gave a clearly better fit than a single path. The split first shell, with an average W-O distance of $1.95 \AA$, is consistent with octahedral coordination in which the $\mathrm{W}$ atom is off-centered, confirming that the dominant W(VI) component sorbed on boehmite is octahedrally coordinated. This finding agrees with W $\mathrm{L}_{1^{-}}$and $\mathrm{L}_{3}$-edge XANES results, showing distorted octahedral coordination for both $\mathrm{pH} 4$ and 8 sorption samples. Based on fits of polymeric tungstate model compounds (Supporting Information), and consistent with the findings of Kuzmin[32], it was necessary to introduce a multiple scattering (MS) contribution to satisfactorily fit the splitting in the first oscillation in the chi functions at $3-3.5 \AA^{-1}$.A four-leg, near linear O-W-O path, based on the configuration in sodium metatungstate, was found to be most suitable. The weak peak at $2.7 \AA$ in the FT (not corrected for phase shift) was alternately fit with aW-Al path and a WW path. The fit with $2.2 \mathrm{Al}$ at $3.14 \AA$ was favored, inasmuch as fits with a W-W path gave a distance $(2.8$ $\AA$ ) considered to be unrealistically short.Furthermore, the intensity of this peak decreases as tungstate concentration increases in the solution.

Fitting of the 200 and $1000 \mu \mathrm{MpH} 8$ samples gave nearly identical fit results. The first peak wasagain best fit with two W-O paths at 1.75 and $2.14 \AA$. A MS path was again found to be necessary, and a W-Al path was fit at 3.14 $\AA$. The peaks near 3.3-3.8 $\AA$ were best fit with two W-W paths at 3.23 and $3.73 \AA$, with combined $\mathrm{CN}$ of $2.2 \pm 0.4$. Fitsfor these peaks were also attempted with $\mathrm{W}-\mathrm{Al}$ paths, 
however, these fits were either not satisfactory or resulted in $\mathrm{W}-\mathrm{Al}$ distances that were not reasonable. The peaks near 3.3-3.8 $\AA$ appear with increasing tungstate concentration, while the peak at $3.1 \AA$ in the 5 $\mu \mathrm{M}$ pH 8 sample diminishes as tungstate concentration increases, indicating that increasing W-W coordination (near 3.3-3.8 )islinked to tungstate concentration.

The $200 \mu \mathrm{M}$ sorption sample at $\mathrm{pH}$ 4was best fit with three W-O paths at $1.73,1.90$ and $2.19 \AA$, and a W-W pathat 3.25 $\mathrm{A}$.In fitting the $200 \mu$ Msorption sample, a second $\mathrm{W}-\mathrm{W}$ path was introduced to fit the right shoulder near 3.3 Åin the FT (not corrected for phase shift), which is similar to the feature in the sorption samples at $\mathrm{pH}$ 8. The $1000 \mu$ Msorption sample was best fit with three W-O paths at 1.74, 1.98, and $2.21 \AA$ A. The coordination number for the first shell of the $200 \mu \mathrm{M}$ and $1000 \mu$ Msamplesat pH 4

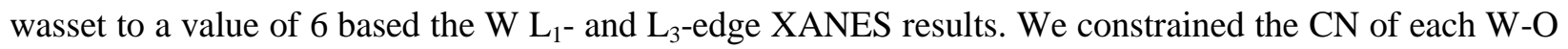
path to 2 based on the W-O paths in model compounds. Fits using two and three W-O paths constrained to total $\mathrm{CN}$ of 6 were compared for fitting the first peak in the FT. Not surprisingly, the use of three W-O pathsgave a better fit to the first oscillation in the chi function and the first peak in the FT, but neither case fit the observed splitting of this oscillation ideally.Fit results were compared with and without a W-Al path, with slightly better results obtained by including W-Al, and especially the small feature near $2.5 \AA$ in the FT (not corrected for phase shift) occurring at the same position of the W-Al path in the $5 \mu \mathrm{M}$ sorption samples. The second shell in the $1000 \mu \mathrm{M}$ pH 4 sample was best fit with a $\mathrm{W}-\mathrm{W}$ path near $3.25 \AA$, consistent with edge-sharing W-W. This peak also increases with increasing tungstate concentration in the solution. We also attempted fits usingadditional W-W paths to explore possible destructive interference resulting from multiple closely spaced W-W backscattering paths. However, this resulted in unrealistic parameters with no clear improvement in fit results. The main difference between the higher concentration $\mathrm{pH} 4$ and $\mathrm{pH} 8$ spectra appears to be a greaterdegree of $\mathrm{W}-\mathrm{W}$ edge-sharing in the pH 4 spectra compared to the $\mathrm{pH} 8$ samples, suggesting some differences in the respective polymeric sorption complexes.

\section{Discussion}




\subsection{Tungstate sorption trends on the boehmite surface}

Tungstate shows a strong affinity for sorbing onto boehmite at low and neutral $\mathrm{pH}$ conditions, with decreasing uptake at higher $\mathrm{pH}$. Observed uptakenear or above the $\mathrm{PZC}$ value of boehmite $(\mathrm{pH}$ 8.6-9.1) suggests thattungstate sorption is not dueentirely to electrostatic attraction. This result is consistent with previous studies investigating the reaction of tungstates with other mineral surfaces[16, 17, 33].The absence of a maximum in uptake of tungstate with increasing concentration in the solution suggests that tungstate sorption on the surface is not limited by the surface site availability over the range studied.We note that a study of the sorption of the $\mathrm{H}_{2} \mathrm{~W}_{12} \mathrm{O}_{40}{ }^{6-}$ polymeric species on boehmite at initial $\mathrm{pH} 5$ observed a sorption maximum reported as $0.08 \mu \mathrm{mol} \mathrm{H}_{2} \mathrm{~W}_{12} \mathrm{O}_{40}{ }^{6-}$ per $\mathrm{m}^{2}$ (equivalent to $0.6 \mathrm{~W} / \mathrm{nm}^{2}$ ) at $\mathrm{H}_{2} \mathrm{~W}_{12} \mathrm{O}_{40}{ }^{6-}$ solution concentrations above $100 \mu \mathrm{M}$ (or 1,200 $\mu \mathrm{M} \mathrm{W}(\mathrm{VI})$ )[15].Direct comparison with our results is complicated bythe different experimental conditions used. Their solution $\mathrm{pH}$ (initially 5) was allowed to drift during uptake, increasing rapidly to $\mathrm{pH} 7$ and decreasing to $\sim 6.3$ at $48 \mathrm{~h}$. They presented spectroscopic evidence suggesting that the $\mathrm{H}_{2} \mathrm{~W}_{12} \mathrm{O}_{40}{ }^{6-}$ polymeric species remains largely intact on sorption. As described below, we are unable to confirm the identity of sorption complexes and consider that multiple species may be present. Nevertheless, it is interesting to compare observed sorption densities with the prior study. For example, the surface coverages fromour $1000 \mu \mathrm{M}$ sorption experiments at $\mathrm{pH} 6$ and 8 (which bracket the final $\mathrm{pH}$ in [15]) are 1.42 and $0.55 \mathrm{~W} / \mathrm{nm}^{2}$, respectively. These values are not greatly different than the coverage observed inthe priorstudy $\left(0.6 \mathrm{~W} / \mathrm{nm}^{2}\right)[15]$.

The desorption experiments show that tungstate uptake is essentially irreversible at $\mathrm{pH} 4$ and only partly reversible at $\mathrm{pH}$ 8. Sorption irreversibility is commonly attributed to formation of a surface precipitate or a stable surface complex, where release of the adsorbate to solution is restricted either because of its greater stability on the solid or due to a kinetic hindrance[34, 35]. Our desorption results imply some differencesin sorption complexes on the boehmite surface at $\mathrm{pH} 4$ and $\mathrm{pH} 8$.

4.2 Tungstate polymerization and coordination change on the boehmite surface 
The most striking finding in our study is the occurrence of polymeric tungstates at the boehmite surface from sorption experiments at both $\mathrm{pH} 4$ and 8, except possibly for the lowest tungstate concentration $(5 \mu \mathrm{M})$. While not surprising for $\mathrm{pH} 4$ sorption samples, this finding was unexpected at $\mathrm{pH}$ 8, where monotungstate dominates in solution. Whereas the XANES signatures of the sorbed polymeric tungstatesare almost indistinguishable between these two $\mathrm{pH}$ conditions, the EXAFS results show small but distinct differences between $\mathrm{pH} 4$ and 8 . The $\mathrm{L}_{1^{-}}$and $\mathrm{L}_{3}$-edge XANES results confirm distorted octahedral coordination of $\mathrm{W}(\mathrm{VI})$ for all sorption samples (Figures5 and 6). The $\mathrm{L}_{3}$-edge EXAFS fits are consistent with such coordination, and also confirm W-W distances that correspond directly to cornerand edge-sharing of $\mathrm{W}(\mathrm{VI})$ octahedra, except for the $5 \mu \mathrm{M}$ sorption samples. These XANES and EXAFS signatures are distinguishing characteristics of polymeric tungstate species.However, because many different polytungstates share these features in various combinations, there is little likelihood of identifying individual species. It is also possible, perhaps even likely, that multiple tungstate species occur on the boehmite surface. Possible supporting evidence may be found in the change of EXAFS results with increasing concentration. ESI-MS results show several types of polytungstates in $\mathrm{pH} 4$ solution, and previous studies have reported various intermediate polytungstate species $[4,5,13]$. Those polymeric tungstates are possible candidates for surface complexes. Yet, the XANES and EXAFS of the sorption samples do not match exactly any particular model compounds that we examined.While the ESIMS results forthe $\mathrm{pH} 4$ solution show monotungstateand several polytungstates, monotungstate is the dominant species in solution at $\mathrm{pH} 8$ (Supporting Information). Our XANES and EXAFS results confirm polytungstates as the major surface complexes.However, it is not possibleto entirely rule out the occurrence of a minor component of tetrahedral tungstate co-existing with polymeric forms on the surface, owing to limited sensitivity of the method.

The occurrence of one or more polymeric tungstate species in the higher concentration sorption samples at $\mathrm{pH} 4$, where polymerization dominates in solution, is not surprising. However, our resultsfor the 200 and $1000 \mu \mathrm{M}$ samples at $\mathrm{pH} 8$ lead us to conclude that one or more polymeric tungstate species 
forms during uptake at the boehmite surface,inasmuch asthe dominant solution species is a monomer. Furthermore, this is accompanied by a change from tetrahedral coordination of W(VI) in solution to octahedral coordination in the polymeric surface complex, a change that also occurs with the $5 \mu \mathrm{M}$ samples. The precise details of how the polymerization and change in coordination occur remain unclear, as does the actual form of the surface species. Yet, interaction of tungstate with the boehmite surface seemingly favorsa coordination change and polymerization. Previous studies of tungstate sorption on iron and manganese oxideshave suggested the existence of monomeric tungstate with octahedral coordination on the surface at $\mathrm{pH} 8[33]$. However, the tungstate concentrations used in that study $(0.15-100 \mu \mathrm{M})$ were restricted to the lower end of the rangein our study $(5-1000 \mu \mathrm{M})$, so thatonly our lowest concentration sample can be compared with their results. Our results areconsistentwith octahedral coordination of $\mathrm{W}(\mathrm{VI})$, and the absence of clear $\mathrm{W}-\mathrm{W}$ backscattering provide plausible evidence for a monomeric tungstate on the surface at this low $\mathrm{W}$ concentration $(5 \mu \mathrm{M})$. However, we cannot entirely rule out the possibility that polymeric tungstate exists on the surface, especially at $\mathrm{pH} 4$ where the polymeric species $\mathrm{H}_{2} \mathrm{~W}_{12} \mathrm{O}_{40}{ }^{6-}$ is dominant in solution.We also note that Clausen et al. reported polytungstate species presentin firing range soilsat $\mathrm{pH}$, with EXAFS data suggestingan $\alpha$-Keggintype cluster[36].We are not aware of any reports of polymeric tungstate sorption complexes at basic $\mathrm{pH}$ conditions.

\subsection{Identification of tungstate species at the surface}

The inability to identify the particular species of sorbed polymeric tungstate(s) because of the similarities they share in coordination and configuration raises interesting questions. First, do multiple species occur at the surface, i.e., more than one type of polymeric unit?This possibilitymight be anticipated at low $\mathrm{pH}$ because of the presence of multiple polytungstate species in solution, some of which may be metastable species associated with slow polymerization kinetics. The subtle changes observed in EXAFS with increasing $\mathrm{W}$ concentration at $\mathrm{pH} 4$ may reflect the appearance of additional surface species at the higher end of the concentration range(Figure8), or possibly related to sorption exceeding available surface sites. Active adsorption site densities on boehmite have been reported for 

In comparison, the surface coverage for our $5 \mu$ Msorption sample at $\mathrm{pH} 4$ is $0.10 \mathrm{~W} / \mathrm{nm}^{2}$, andthose for the 200 and $1000 \mu \mathrm{M}$ samples are 0.87 , and $2.47 \mathrm{~W} / \mathrm{nm}^{2}$, respectively. These latter surface coverages likely exceed availableadsorption sites.

A related question is whether the differences in sorption reversibility between $\mathrm{pH} 4$ and 8 can be explained by different surface complexes. Sorption at $\mathrm{pH} 4$ is largely irreversible, whereas at $\mathrm{pH} 8$ some reversibility $(25-30 \%)$ is observed (Figure 4). The EXAFS results reveal differences in the W-W paths between $\mathrm{pH} 4$ and $\mathrm{pH} 8$ samples (for both 200 and $1000 \mu \mathrm{M}$ ), suggesting structural differences in the surface complexes, which could be responsible for the differing desorption behaviors. The presence of multiple surface species at one of the $\mathrm{pH}$ conditions could also influence the observed behavior. The $\mathrm{pH}$ may also play somerole in controlling the observed sorption reversibility, as suggested by polymerization behavior in solution. At low $\mathrm{pH}$ polymerization of $\mathrm{W}(\mathrm{VI})$ is clearly favored, whereas at near neutral and higher $\mathrm{pH}$ monomeric tungstate is favored. Hence, one could speculate that the polymeric surface complexes are more stable at $\mathrm{pH} 4$ than at $\mathrm{pH}$ 8. This would be consistent with irreversible sorption at the lower $\mathrm{pH}$ but with some limited degree of reversibility at the higher $\mathrm{pH}$. The effect of $\mathrm{pH}$ on surface charge may also play a role in sorption reversibility. The more positively charged surface at $\mathrm{pH} 4$ would create more favorable circumstances forsorbedpolytungstates, which have large negative charges.

It is interesting to consider whether formation of polymeric tungstates at the surface is analogous to surface precipitation.In both cases, W(VI) forms extended structures at the surface. We consider that no distinction between them would be possible using EXAFS.

\subsection{Comparison with molybdate sorption on (hydr)oxide surfaces}

In view of the similarities noted between tungstate and molybdate systems, it is interesting to compare their sorption behavior.Molybdate shows a distribution of species in solution broadly similar to tungstate, favoringmonomeric species at neutral and high $\mathrm{pH}$ and formation of polymeric species at low $\mathrm{pH}[11]$.Arai investigated molybdate species sorbedon the goethite $(\mathrm{FeOOH})$ surface using Mo K-edge 
EXAFS[37]. This study reported the existence of monomeric tetrahedral molybdate at the surface at near neutral $\mathrm{pH}$ and a mixture of monomeric tetrahedral and polymeric octahedral molybdate at acidic $\mathrm{pH}$. Increasing Mo loading favored the formation of the polymeric form. Wasylenki et al. used Mo K-edge EXAFS to investigate molybdate species sorbedon birnessite $\left(\mathrm{MnO}_{2}\right)[38]$.In sorption experiments performed at $\mathrm{pH}$ 8.0-8.5, where monomeric tetrahedral molybdate is the dominant solution species, they found the sorbed species to be a polymeric molybdate with distorted octahedral coordination. This finding differs from the results of Arai at near neutral $\mathrm{pH}$, where monomeric tetrahedral molybdate was reported. This difference may reflect the differing properties of the sorbent phases and/or their surface charge. However, both studies identified the tendency for molybdate to polymerize when sorbed at metal (hydr)oxide surface, although at different $\mathrm{pH}$ conditions. In our study, we find that tungstate exhibits a strong tendency to form a polymeric species on the surface of boehmite over a wide range of $\mathrm{pH}$ and tungstate concentrations. Only at the lowest concentration $(5 \mu \mathrm{M})$, do we find evidence for a monomeric octahedral complex. At $\mathrm{pH} 8$, sorption is accompanied by a change in $\mathrm{W}(\mathrm{VI})$ coordination from tetrahedral (in solution) to distorted octahedral (on the surface).The tendency that we observe for polymerization of tungstate over the entire $\mathrm{pH}$ range 4-8 may reflect the different sorbent (boehmite) and/or a greater inherent tendency for tungstate to polymerize compared to molybdate. Further studies of tungstate sorption on different mineral surfaces are needed to evaluate the importance of surface-driven tungstate polymerization.

4.5 Possible mechanisms for tungstate polymerization and coordination change on the boehmite surface

The coordination change and the polymerization of tungstate sorbed on boehmite at $\mathrm{pH} 8$ were observed by XANES and EXAFS in our study. However, the mechanisms still remain unclear. We speculate that formation of an inner-sphere surface complex could induce the observed symmetry change from tetrahedral $\mathrm{WO}_{4}{ }^{2-}$ to octahedral coordination, with the addition of water molecules. Like other tetrahedral oxyanions (e.g., $\mathrm{PO}_{4}{ }^{3-}$ ), we presume that tetrahedral tungstate would initially form a bidentate 
binuclear surface complex on the boehmite surface at $\mathrm{pH} 8$ [27]. Our EXAFS fit results for the $5 \mu \mathrm{M}$ sorption sample at $\mathrm{pH} 8$ support formation of an inner-sphere, octahedral complex, showing a W-Al path at $3.14 \AA$. The $\mathrm{W}-\mathrm{O}$ bonds for the oxygen atoms bridging with $\mathrm{Al}$ atoms at the surface would lengthen, thereby lowering their bond strengths and also allowing introduction of water molecules that ultimately result in the change to octahedral coordination (Figure S4).Previous XANES and EXAFS studies of tungstate species deposited on prepared $\mathrm{Al}_{2} \mathrm{O}_{3}$ surfacesobserved the coordination change of tetrahedral tungstate to octahedral dependent on the availability of water molecules at the surface[39, 40].This modelsuggests that the sorption mode(s) of tungstate on the surfaces playsan important role in the coordination change.

Similar results were observed in previous studies of molybdate species on various oxide catalyst surfaces, such as $\delta-\mathrm{MnO}_{2}, \mathrm{TiO}_{2}$, ferrihydrite, $\mathrm{Al}_{2} \mathrm{O}_{3}$, goethite, and hematite [41-46].In these studies, molybdates forming inner-sphere complexes on $\delta-\mathrm{MnO}_{2}, \mathrm{TiO}_{2}$, andhematite were found to have octahedral symmetry, while tetrahedral molybdates found on ferrihydrite and $\mathrm{Al}_{2} \mathrm{O}_{3}$ surfaces were interpreted to form outer-sphere complexes [47].The different coordination geometries of Mo on Fe (oxyhydr)oxides imply that specific properties of the surface influence the coordination change and that the coordination change was induced by the inner-sphere surface complexation of Mo in these studies. Further studies of tungstate sorption on various mineral and oxide surfaces are needed to reveal whether similar relationships exist between the properties of the mineral surface and the coordination of adsorbed tungstate species.

We can envision two possible surface polymerization mechanismsto explain our observations at $\mathrm{pH}$ 8: (1) the polymerization of neighboring tungstate species sorbed on the surface and (2) additive polymerization with a tetrahedral complex in a terminal position. Schematic models for these mechanisms are shown in Figure 9. The former should be dependent on a sufficiently high surface coverage of tungstate to allow linkages that result in edge- or corner-sharing of tungstate octahedra. We note that this mechanism may not be consistent with the continued tungstate uptake that we observed in the isotherm 
results, sincethis model requires that polymerization occurs only between tungstate groups sorbed on the surface.

The second model is an example of open-chain polymerization and is similar to the tungstate polymerization mechanism suggested by Walanda et al. in solution based on ESI-MS results[13].Monotungstate surface complexes, which would be octahedral as described above, could act as preferred sites for attachment of tetrahedral tungstate species in solution. Hence, the terminal tetrahedral tungstate is available to form tungstate chains with additional monomers. Additional monomers share two oxygen atoms with the terminal tungstate (tetrahedral), resulting in the coordination change to octahedral. Once the seeds have been formed by the surface sorption, polymerization is driven by the addition of monomers. This model allows continued attachment from solution, and is therefore consistent with the isotherm results. With the present information, we cannot determine whether either of these possible mechanisms of tungstate polymerization isrelevant in our experiments. Further studies using complementary approaches are needed to understand these mechanisms better.

4.6 Possible environmental implications of tungstate polymerization

Strigul reported that speciationof tungstate is likely to be important forcontrolling its mobility, toxicity, and bioavailability in aquatic systems[1]. Our present findingsdemonstrate that tungstate forms stable polymeric surface complexes when sorbed on boehmite over a range of $\mathrm{pH}$ conditions. Hence the mobility of dissolved tungstate may be effectively limited by sorption on surfaces of (hydr)oxides, such asboehmite. However, we still lack an understanding of the toxicity and bioavailability of polytungstates bound on the surface of fine mineral particles in the environment. Evaluation of the toxicity ofpolytungstatessorbed on other mineral surfaces will be important for a complete understanding of the detailed environmental behavior of tungstate.

\section{Conclusions}


In this study, we examined the systematics and molecular-scale mechanisms of tungstate sorption onto boehmite over a range of $\mathrm{pH}$ values and tungstate concentrations. Batch uptake results reveal sorption behavior that is expected for anions, with tungstate binding strongly and irreversiblyat low $\mathrm{pH}$ and less strongly with increasing $\mathrm{pH}$. XAS analysis confirmsthe presence of polytungstate complexes on the surface over the $\mathrm{pH}$ range 4-8, although the exact tungstate species cannot be determined. Polymerization of tungstate appears to be favored during sorption onto boehmite. At $\mathrm{pH} 8$, where monotungstate is the dominant solution species, polymerization is accompanied by a change in coordination from tetrahedral (in solution) to octahedral in the surface complex. The results suggest that sorption onto boehmtie is an effective means for environmental remediation of dissolved tungstate. The role of the mineral surface in favoring polymerization of tungstate remains unclear, and further studies should be undertaken using different sorbent phases. The results of this study are relevantfor understanding tungstate mobility and bioavailability, which are strongly influenced by its sorption behavior, and should be accounted for in models concerned with the solubility, mobility, and accumulation of tungsten species in natural environments.

\section{Acknowledgements}

We thank Robert Riegerof the Stony Brook University Proteomics Center for assisting with ESIMS data collection. The boehmite used in this study was provided by Dr. William Casey (UC Davis). This research was supported by the National Science Foundation, Grant CHE0714183. We also thank the beamline scientists at X11A, NSLS, and 12BM and 20BM, APSfor their assistance with XAS measurements. Use of the National Synchrotron Light Source, Brookhaven National Laboratory, was supported by the U.S. Department of Energy, Office of Science, Office of Basic Energy Sciences, under Contract No. DE-AC02-98CH10886. Use of the Advanced Photon Source, an Office of Science User Facility operated for the U.S. Department of Energy (DOE) Office of Science by Argonne National 
Laboratory, was supported by the U.S. DOE under Contract No. DE-AC02-06CH11357. Comments from two anonymous reviewers improved the manuscript. 


\section{References}

1. Strigul, N., Does speciation matter for tungsten ecotoxicology? Ecotoxicol. Environ. Safety, 73, (6), 1099-1113 (2010).

2. Clausen, J. L.; Korte, N., Environmental fate of tungsten from military use. Sci. Total Environ., 407, (8), 2887-2893(2009)

3. Bednar, A. J.; Jones, W. T.; Boyd, R. E.; Ringelberg, D. B.; Larson, S. L., Geochemical parameters influencing tungsten mobility in soils. J. Environ. Qual., 37, (1), 229-233 (2008)

4. Smith, B. J.; Patrick, V. A., Quantitative determination of sodium metatungstate speciation by W183 NMR spectroscopy. Aust. J. Chem., 53, (11-12), 965-970 (2000)

5. Hastings, J. J.; Howarth, O. W., $\mathrm{A}^{183} \mathrm{~W},{ }^{1} \mathrm{H}$ and ${ }^{17} \mathrm{O}$ nuclear-magnetic-resonance study of aqueous isopolytungstates. J. Chem. Soc. Dalton Trans., (2), 209-215 (1992)

6. Cruywagen, J. J.; vander Merwe, I. F. J., Tungsten(VI) equilibria:Apotentiometric and calorimetric investigation. J. Chem.Soc. Dalton Trans., (7), 1701-1705 (1987)

7. Dermatas, D.; Braida, W.; Christodoulatos, C.; Strigul, N.; Panikov, N.; Los, M.; Larson, S., Solubility, sorption, and soil respiration effects of tungsten and tungsten alloys. Environ. Forensics, 5, (1), 5-13 (2004)

8. Strigul, N.; Koutsospyros, A.; Arienti, P.; Christodoulatos, C.; Dermatas, D.; Braida, W., Effects of tungsten on environmental systems. Chemosphere, 61, (2), 248-258(2005)

9. $\quad$ Ringelberg, D. B.; Reynolds, C. M.; Winfield, L. E.; Inouye, L. S.; Johnson, D. R.; Bednar, A. J., Tungsten effects on microbial community structure and activity in a soil. J. Environ. Qual., 38, (1), 103-110 (2009)

10. Strigul, N.; Koutsospyros, A.; Christodoulatos, C., Tungsten speciation and toxicity: Acute toxicity of mono- and poly-tungstates to fish. Ecotoxicol. Environ. Safety, 73, (2), 164-171 (2010)

11. Cruywagen, J. J., Protonation, oligimerization, and condensation reactions of vanadate(V), molybdate(VI), and tungstate(VI). Adv. Inorg. Chem., 49, 127-182 (2000)

12. Fierro, J., Metal oxides: Chemistry and applications. CRC press: 2006; vol. 108. 
13. Walanda, D. K.; Burns, R. C.; Lawrance, G. A.; von Nagy-Felsobuki, E. I., Electrospray mass spectrometry of aqueous solutions of isopolyoxotungstates. J. Cluster Sci., 11, (1), 5-28 (2000)

14. Aveston, J., Hydrolysis of tungsten(VI): Ultracentrifugation acidity measurements, and Raman spectra of polytungstates. Inorg. Chem.,3, (7), 981-986(1964)

15. Hernandez, J., Synthese de nanoparticulesd'oxydes de feretd'aluminium pour l'etude del'adsorptiond'entitesinorganiquespolycondensees. These de Doctorat de l'Universite Pierre et Marie Curie, Paris, 1998.

16. Xu, N.; Christodoulatos, C.; Braida, W., Modeling the competitive effect of phosphate, sulfate, silicate, and tungstate anions on the adsorption of molybdate onto goethite. Chemosphere, 64, (8), 1325-1333(2006)

17. Gustafsson, J. P., Modelling molybdate and tungstate adsorption to ferrihydrite. Chem. Geol., 200, (1-2), 105-115(2003)

18. Sugiyama, S.; Kanda, Y.; Ishizuka, H.; Sotowa, K., Removal and regeneration of aqueous divalent cations by boehmite. J. Colloid Interface Sci., 320, (2), 535-539 (2008)

19. Li, W.; Feng, J.; Kwon, K. D.; Kubicki, J. D.; Phillips, B. L., Surface speciation of phosphate on boehmite ( $\gamma$-AlOOH) determined from NMR spectroscopy. Langmuir, 26, (7), 4753-4761 (2010)

20. Strathmann, T. J.; Myneni, S. C. B., Effect of soil fulvic acid on nickel(II) sorption and bonding at the aqueous-boehmite $(\gamma-\mathrm{AlOOH})$ interface. Environ. Sci. Technol., 39, (11), 4027-4034 (2005)

21. Granados-Correa, F.; Jimenez-Becerril, J., Chromium (VI) adsorption on boehmite. J. Hazard.Mater., 162, (2-3), 1178-1184 (2009)

22. Nordin, J.; Persson, P.; Laiti, E.; Sjoberg, S., Adsorption of o-phthalate at the water-boehmite $(\gamma-$ $\mathrm{AlOOH}$ ) interface: Evidence for two coordination modes. Langmuir, 13, (15), 4085-4093 (1997)

23. Su, C.; Suarez, D. L., In situ infrared speciation of adsorbed carbonate on aluminum and iron oxides. Clays Clay Mineral., 45, (6), 814-825 (1997)

24. Newville, M., IFEFFIT: interactive XAFS analysis and FEFF fitting. J. Synchrotron Rad., 8, 322$324(2001)$ 
25. Ressler, T., WinXAS: A new software package not only for the analysis of energy-dispersive XAS data. J. Phys. IV, 7, (C2), 269-270(1997)

26. Zabinsky, S. I.; Rehr, J. J.; Ankudinov, A.; Albers, R. C.; Eller, M. J., Multiple-scattering calculations of X-ray absorption spectra. Phys. Rev. B, 52, (4), 2995-3009(1995)

27. Li, W.; Feng, X. H.; Yan, Y. P.; Sparks, D. L.; Phillips, B. L., Solid-state NMR spectroscopic study of phosphate sorption mechanisms on aluminum (hydr)oxides. Environ. Sci. Technol., 47, (15), 8308-8315(2013)

28. van Geen,A.; Robertson, A. P.; Leckie, J. O., Complexation of carbonate species at the goethite surface: Implications for adsorption of metal ions in natural waters.Geochim. Cosmochim. Acta, 58, (9), 2073-2086(1994)

29. Villalobos,M.; Leckie, J. O., Carbonate adsorption on goethite under closed and open $\mathrm{CO}_{2}$ conditions.Geochim. Cosmochim. Acta, 64, (22), 3787-3802(2000)

30. Yamazoe, S.; Hitomi, Y.; Shishido, T.; Tanaka, T., XAFS study of tungsten $\mathrm{L}_{1^{-}}$and $\mathrm{L}_{3}$-edges: Structural analysis of $\mathrm{WO}_{3}$ species loaded on $\mathrm{TiO}_{2}$ as a catalyst for photo-oxidation of $\mathrm{NH}_{3}$. J. Phys. Chem. C, 112, (17), 6869-6879 (2008)

31. Kuzmin, A.; Purans, J., Local atomic and electronic structure of tungsten ions in $\mathrm{AWO}_{4}$ crystals of scheelite and wolframite types. Radiation Measurements, 33, (5), 583-586 (2001)

32. Kuzmin, A.; Purans, J.; Benfatto, M.; Natoli, C. R., X-ray-absorption study of rhenium $\mathrm{L}_{3}$ and $\mathrm{L}_{1}$ edges in $\mathrm{ReO}_{3}$ :Multiple-scattering approach. Phys. Rev. B, 47, (5), 2480-2486 (1993)

33. Kashiwabara, T.; Takahashi, Y.; Marcus, M. A.; Uruga, T.; Tanida, H.; Terada, Y.; Usui, A., Tungsten species in natural ferromanganese oxides related to its different behavior from molybdenum in oxic ocean. Geochim.Cosmochim.Acta, 106, 364-378 (2013)

34. Tang, Y.; Reeder, R. J., Enhanced uranium sorption on aluminum oxide pretreated with arsenate. Part I: Batch uptake behavior. Environ. Sci. Technol., 43, (12), 4446-4451 (2009)

35. Tang, Y.; McDonald, J.; Reeder, R. J., Enhanced uranium sorption on aluminum oxide pretreated with arsenate. Part II: Spectroscopic studies. Environ. Sci. Technol., 43, (12), 4452-4458 (2009) 
36. Clausen, J. L.; Bostick, B. C.; Bednar, A.; Sun, J.; Landis, J. D. Tungsten speciation in firing range soils.U.S. Army Corps of Engineers, Engineer Research and Development Center TR-11$1(2011)$

37. Arai, Y., X-ray absorption spectroscopic investigation of molybdenum multinuclear sorption mechanism at the goethite-water interface. Environ. Sci. Technol., 44, (22), 8491-8496 (2010)

38. Wasylenki, L. E.; Weeks, C. L.; Bargar, J. R.; Spiro, T. G.; Hein, J. R.; Anbar, A. D., The molecular mechanism of Mo isotope fractionation during adsorption to birnessite. Geochim.Cosmochim.Acta, 75, (17), 5019-5031 (2011)

39. Hilbrig, F.; Gobel, H. E.; Knozinger, H.; Schmelz, H.; Lengeler, B., X-ray absorption spectroscopy study of the titania- and alumina-supported tungsten oxide system. J. Phys. Chem., 95, (18), 6973-6978 (1991)

40. Horsley, J. A.; Wachs, I. E.; Brown, J. M.; Via, G. H.; Hardcastle, F. D., Structure of surface tungsten oxide species in the $\mathrm{WO}_{3} / \mathrm{Al}_{2} \mathrm{O}_{3}$ supported oxide system from X-ray absorption near-edge spectroscopy and Raman spectroscopy. J. Phys. Chem., 91, (15), 4014-4020 (1987)

41. Mensch, C. T. J.; Vanveen, J. A. R.; Vanwingerden, B.; Vandijk, M. P., Extended X-ray absorption fine-structure study of $\mathrm{Mo} / \mathrm{Al}_{2} \mathrm{O}_{3}$ samples prepared by equilibrium adsorption of ammonium heptamolybdate. J. Phys. Chem., 92, (17), 4961-4964 (1988)

42. Bare, S. R.; Mitchell, G. E.; Maj, J. J.; Vrieland, G. E.; Gland, J. L., Local site symmetry of dispersed molybdenum oxide catalysts: XANES at the Mo $\mathrm{L}_{2,3}$-edges. J. Phys. Chem., 97, (22), 6048-6053 (1993)

43. Aritani, H.; Tanaka, T.; Funabiki, T.; Yoshida, S.; Eda, K.; Sotani, N.; Kudo, M.; Hasegawa, S., Study of the local structure of molybdenum-magnesium binary oxides by means of $\mathrm{Mo} \mathrm{L}_{3}$-edge XANES and UV-vis spectroscopy. J. Phys. Chem., 100, (50), 19495-19501 (1996)

44. Takenaka, S.; Tanaka, T.; Funabiki, T.; Yoshida, S., Structures of molybdenum species in silicasupported molybdenum oxide and alkali-ion-modified silica-supported molybdenum oxide. J. Phys. Chem. B, 102, (16), 2960-2969 (1998)

45. Radhakrishnan, R.; Reed, C.; Oyama, S. T.; Seman, M.; Kondo, J. N.; Domen, K.; Ohminami, Y.; Asakura, K., Variability in the structure of supported $\mathrm{MoO}_{3}$ catalysts: Studies using Raman and 
X-ray absorption spectroscopy with ab initio calculations. J. Phys. Chem. B, 105, (36), 8519-8530 (2001)

46. Lede, E. J.; Requejo, F. G.; Pawelec, B.; Fierro, J. L. G., XANES Mo L-edges and XPS study of Mo loaded in HY zeolite. J. Phys. Chem. B, 106, (32), 7824-7831 (2002)

47. Kashiwabara, T.; Takahashi, Y.; Tanimizu, M.; Usui, A., Molecular-scale mechanisms of distribution and isotopic fractionation of molybdenum between seawater and ferromanganese oxides. Geochim.Cosmochim.Acta, 75, (19), 5762-5784 (2011)

\section{List of figure captions}

Figure 1.Aqueous tungstate speciation at total tungstate concentrations of 5 and $1000 \mu \mathrm{M}$, calculated using the program PHREEQC with stability constants reported in Table S1 (Supporting Information) at ionic strength 0.01 M.Polymerization is favored with increasing tungstate concentration. Species present at low concentrations are not labeled.

Figure 2. Comparison of tungstate sorbed ontoboehmite at different ionic strength conditions as a function of $\mathrm{pH}$ for $50 \mu \mathrm{M}$ total tungstate.

Figure 3.Sorptionisotherms of tungstate on boehmiteas a function of $\mathrm{pH}$ at room temperature. No maximum in sorption is observed over the concentration range studied.

Figure4. Tungstate desorption from boehmite at $\mathrm{pH} 4$ (squares) and 8 (circles) as a function of time, at 200 and $400 \mu \mathrm{M}$ total tungstate.

Figure 5.Tungsten $\mathrm{L}_{3}$-edge XANES spectra (a) and correspondingsecond derivative spectra (b) for selected model compounds showing differences that distinguish tetrahedral $(\mathrm{T})$ and octahedral $(\mathrm{O})$ coordination of $\mathrm{W}(\mathrm{VI})$. Spectra for $5 \mu \mathrm{M}$ sorption samples are shown at top for $\mathrm{pH} 4$ and 8. 
Figure6. Relationship between the splitting of the minima in the $\mathrm{L}_{3}$-edge XANES second derivatives and the pre-edge peak area (a.u.) of $\mathrm{L}_{1}$-edge XANES. a) $\mathrm{Na}_{2} \mathrm{WO}_{4} \cdot 2 \mathrm{H}_{2} \mathrm{O}$, b) $\mathrm{CaWO}_{4}$, c) phosphotungstic acid, d) $\mathrm{WO}_{3}$, e) $\mathrm{Na}_{2} \mathrm{~W}_{2} \mathrm{O}_{7}$, f) sodium metatungstate, g) $\mathrm{Ba}_{2} \mathrm{NiWO}_{6}$, h) $\mathrm{TBA}-\mathrm{W}_{6} \mathrm{O}_{19}$. All sorption samples are tightly clustered (yellow circle). Sorption samples are summarized in Supporting Information (Table S2)

Figure 7.Tungsten $\mathrm{L}_{3}$-edge EXAFS chi functions (left) and corresponding Fourier transform magnitudes (right, not corrected forphase shifts) for $1 \mathrm{mM}$ tungstate solutions ( $\mathrm{pH} 4$ and 8 ) and selected model compounds. Schematic representations show typical W-W distances associated with edge- and cornersharing of tungstate octahedra.

Figure 8.Tungsten $\mathrm{L}_{3}$-edge EXAFS chi functions (left) and Fourier transformation magnitudes (right) for tungstate sorbed on boehmite. Solid lines are data and dashed lines are fits. Arrows indicate the primary differences between high concentration $\mathrm{pH} 4$ and $\mathrm{pH} 8$ sorption samples.

Figure 9. Possible models for tungstate polymerization on the boehmite surface. The polymerization of neighboring tungstate surface complexes is shown in a. The open chain polymerization mechanism is depicted in $b$. 

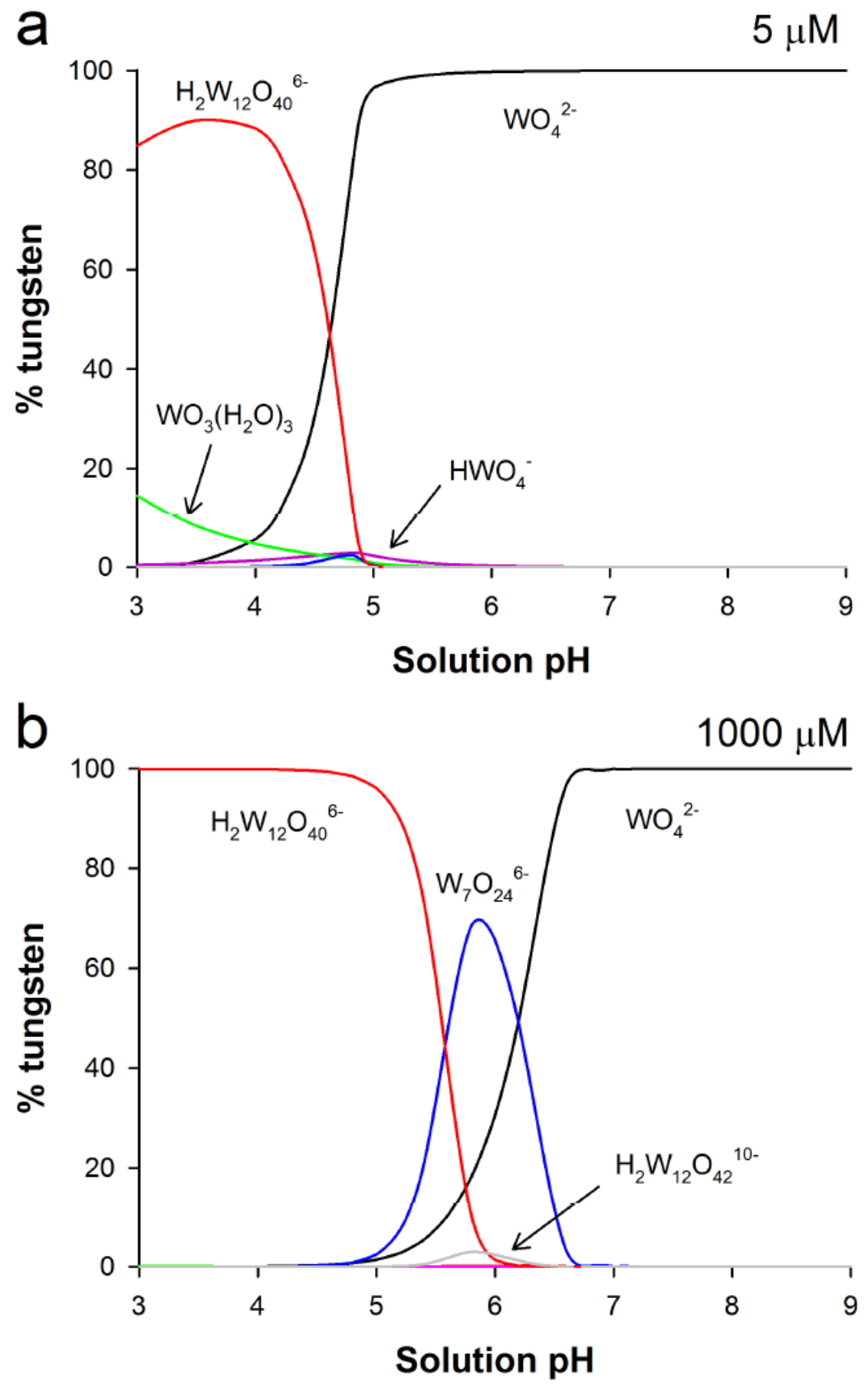
Figure 1.

7

8

9

10

11

12

13

14

15

16

17

18

19

20

21

22

23

24

25

26

27

28

29

30

31

32

33

34

35

36

37

38

39

40

41

42

43

44

45

46

47

48

49

50

51

52

53

54

55

56

57

58

59

60

61

62

63

64

65 


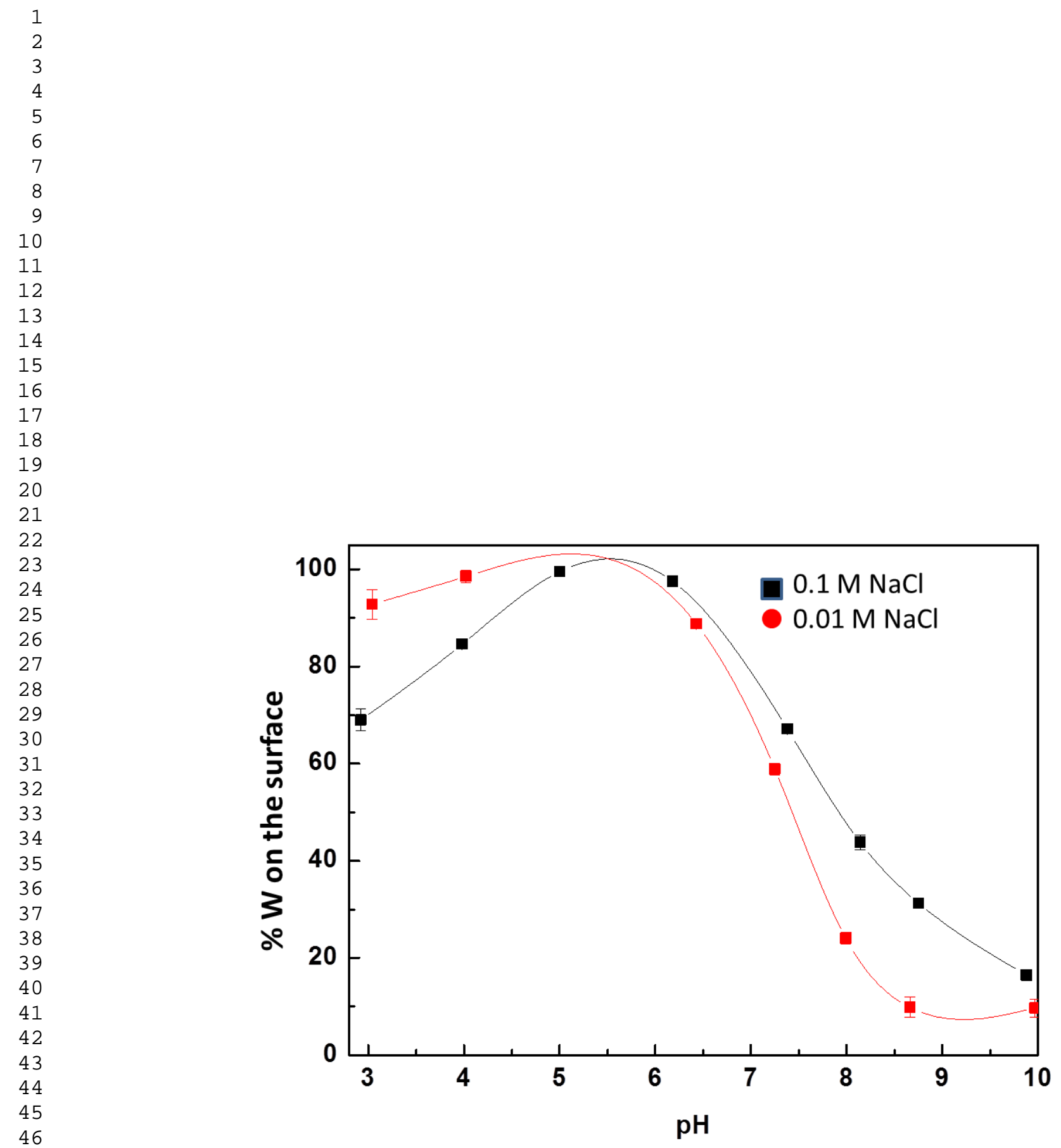

Figure2. 


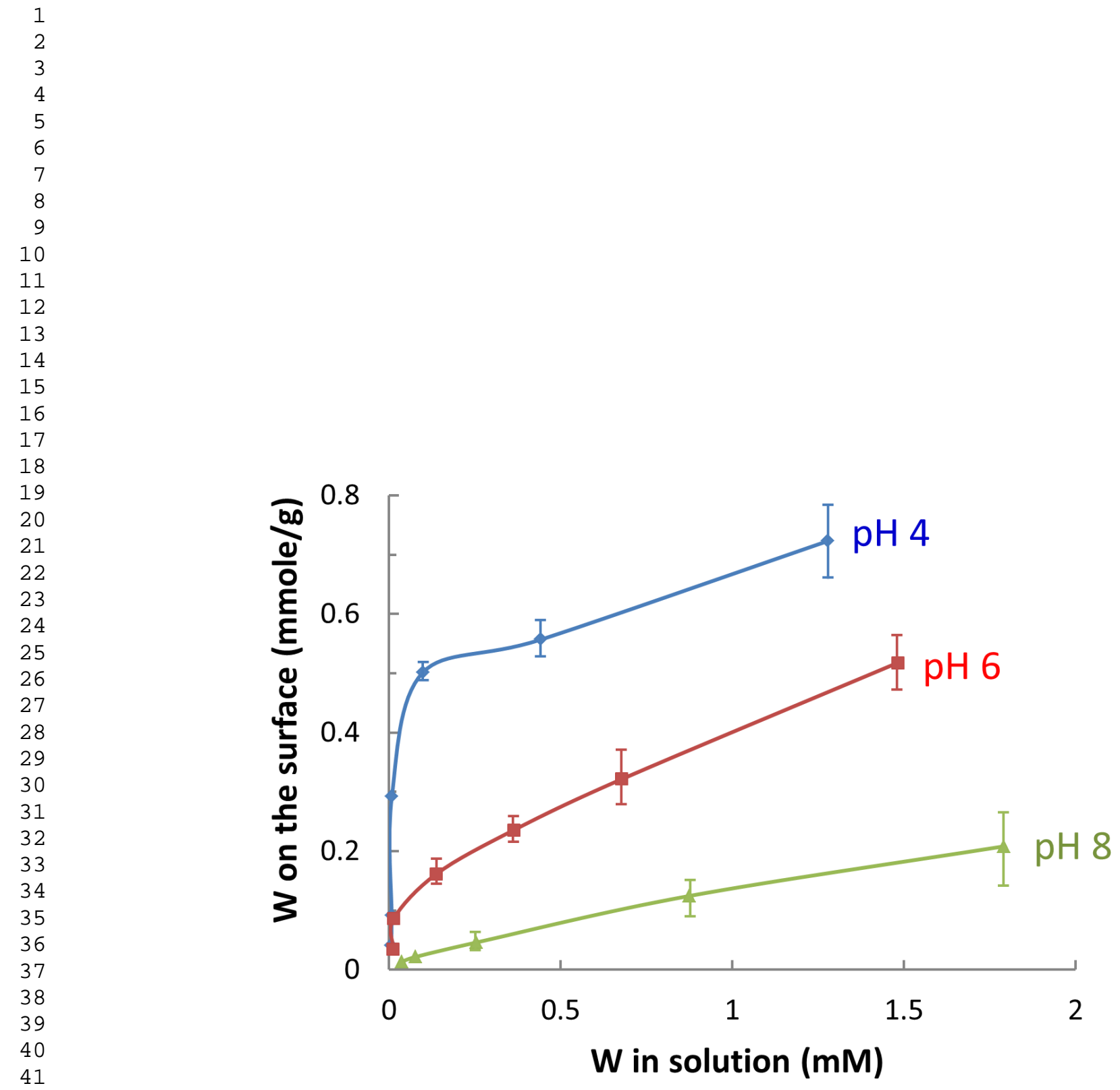

Figure3. 

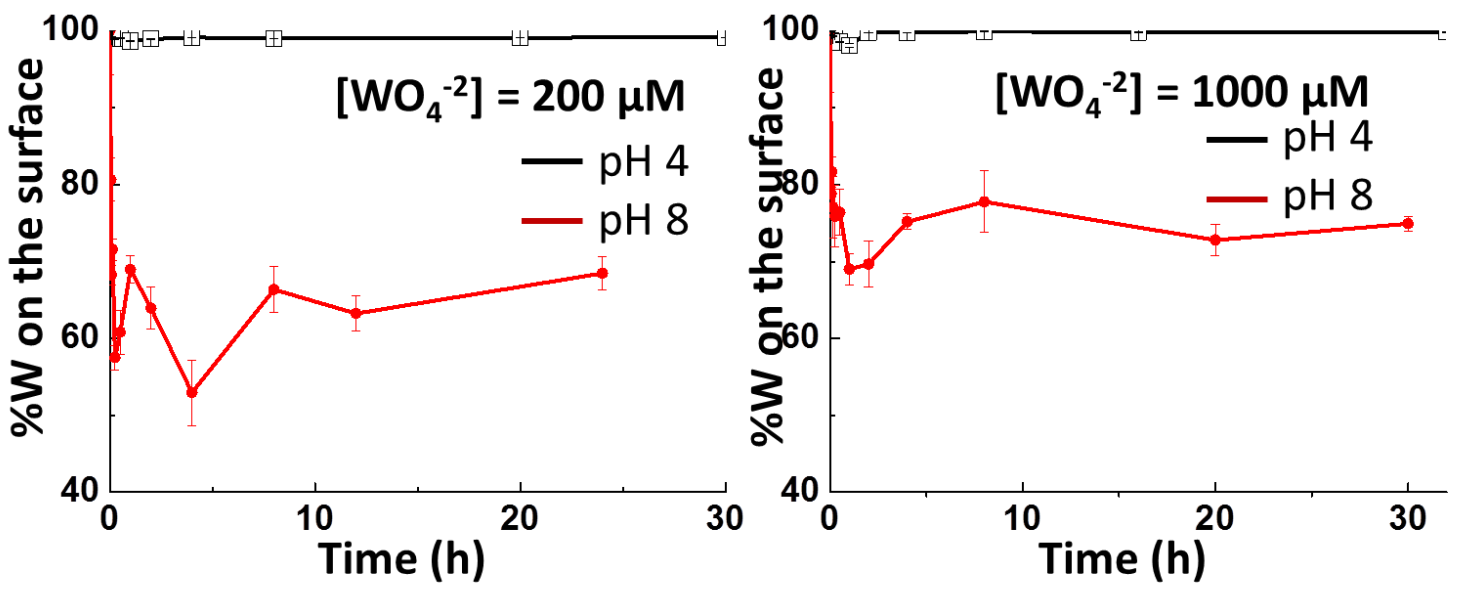

Figure4. 


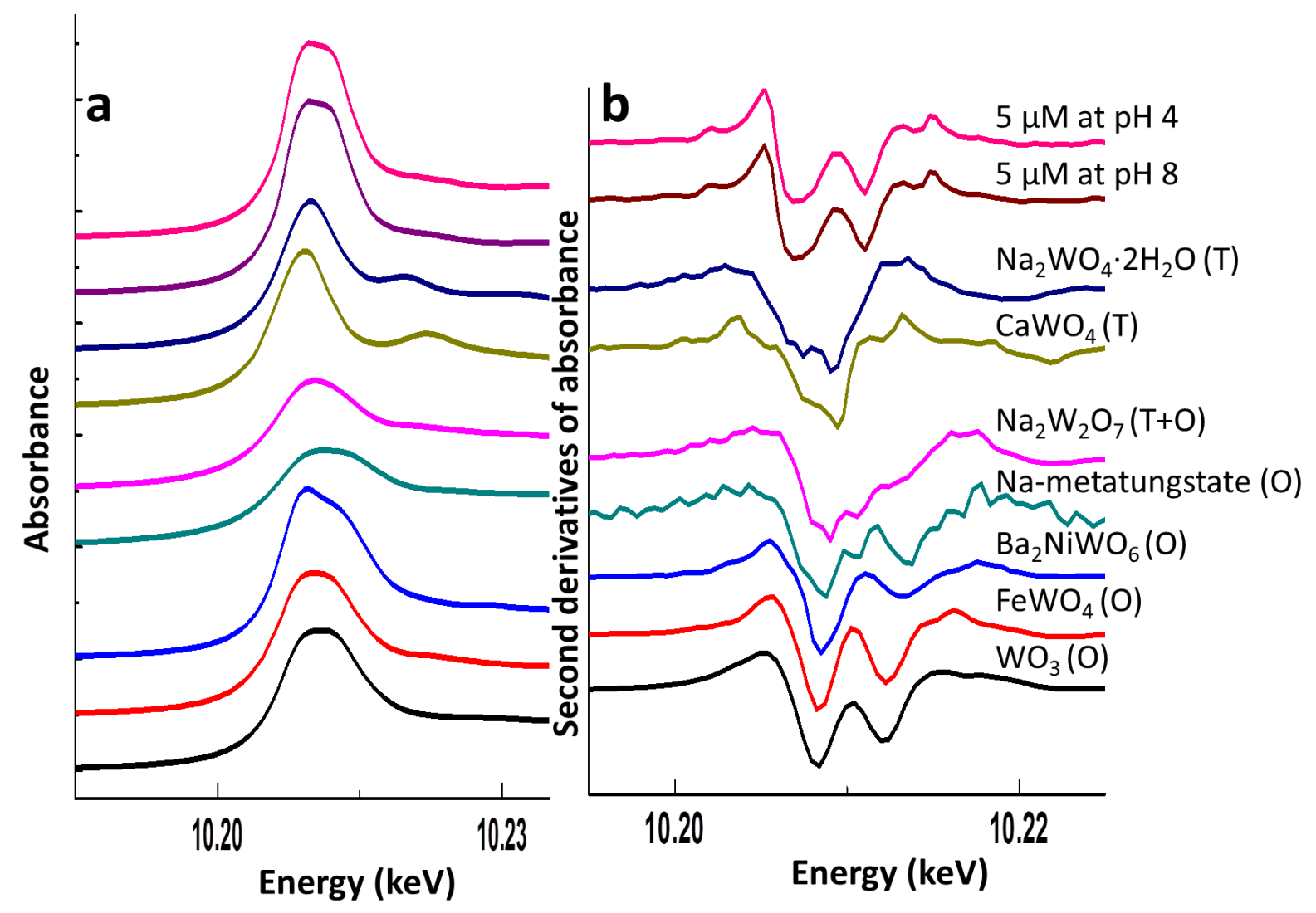

Figure5. 


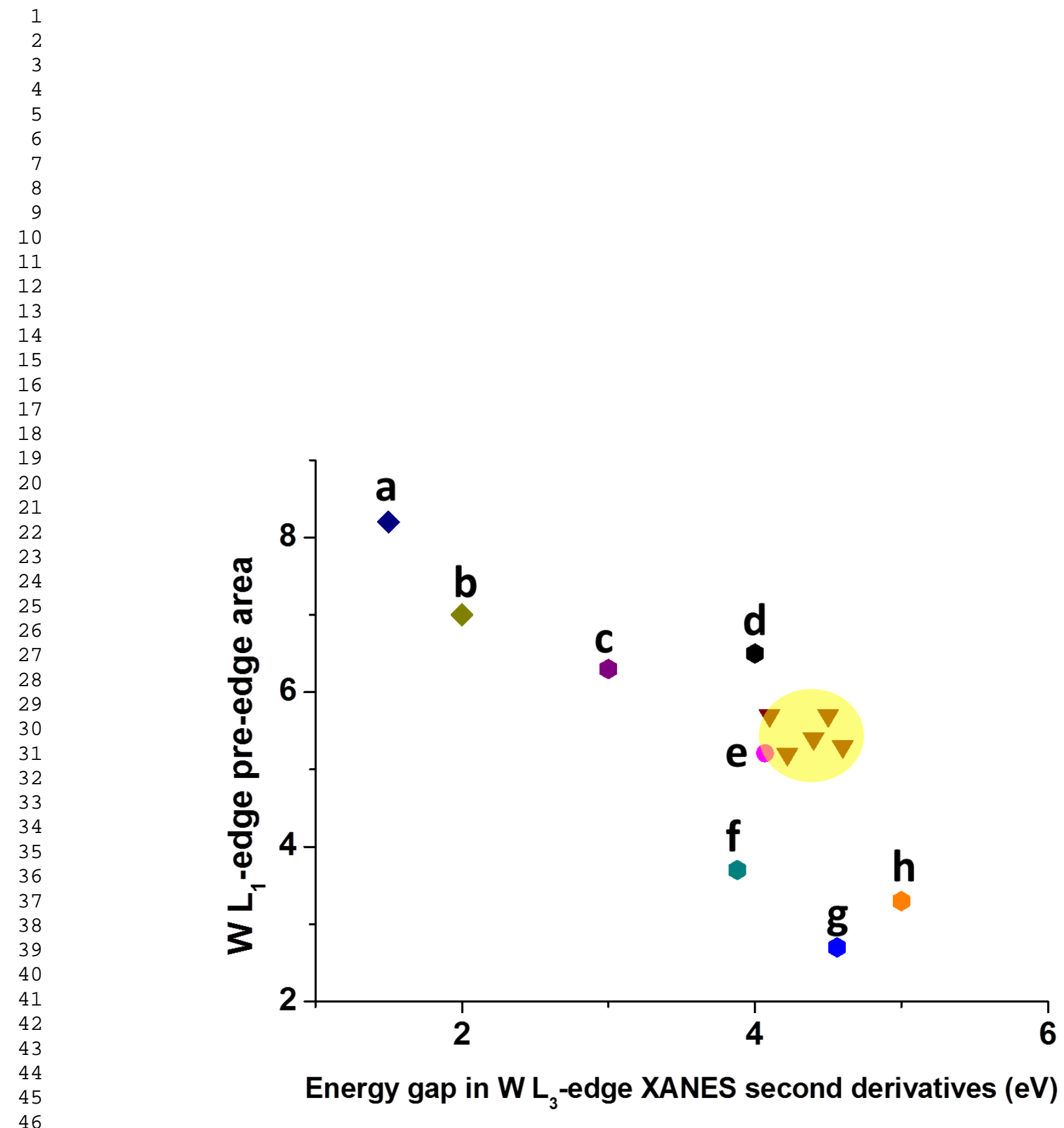

Figure6. 


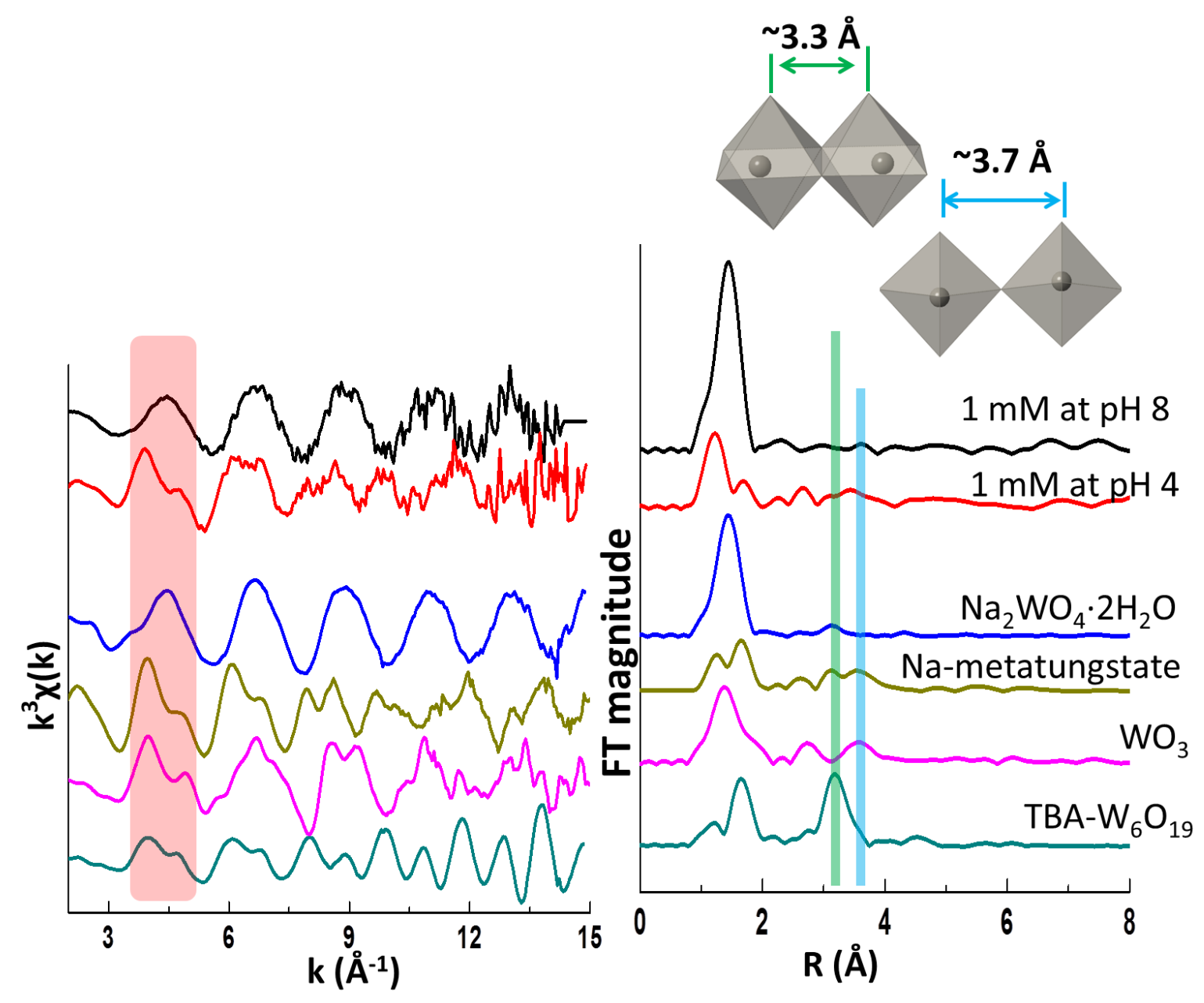

Figure 7. 

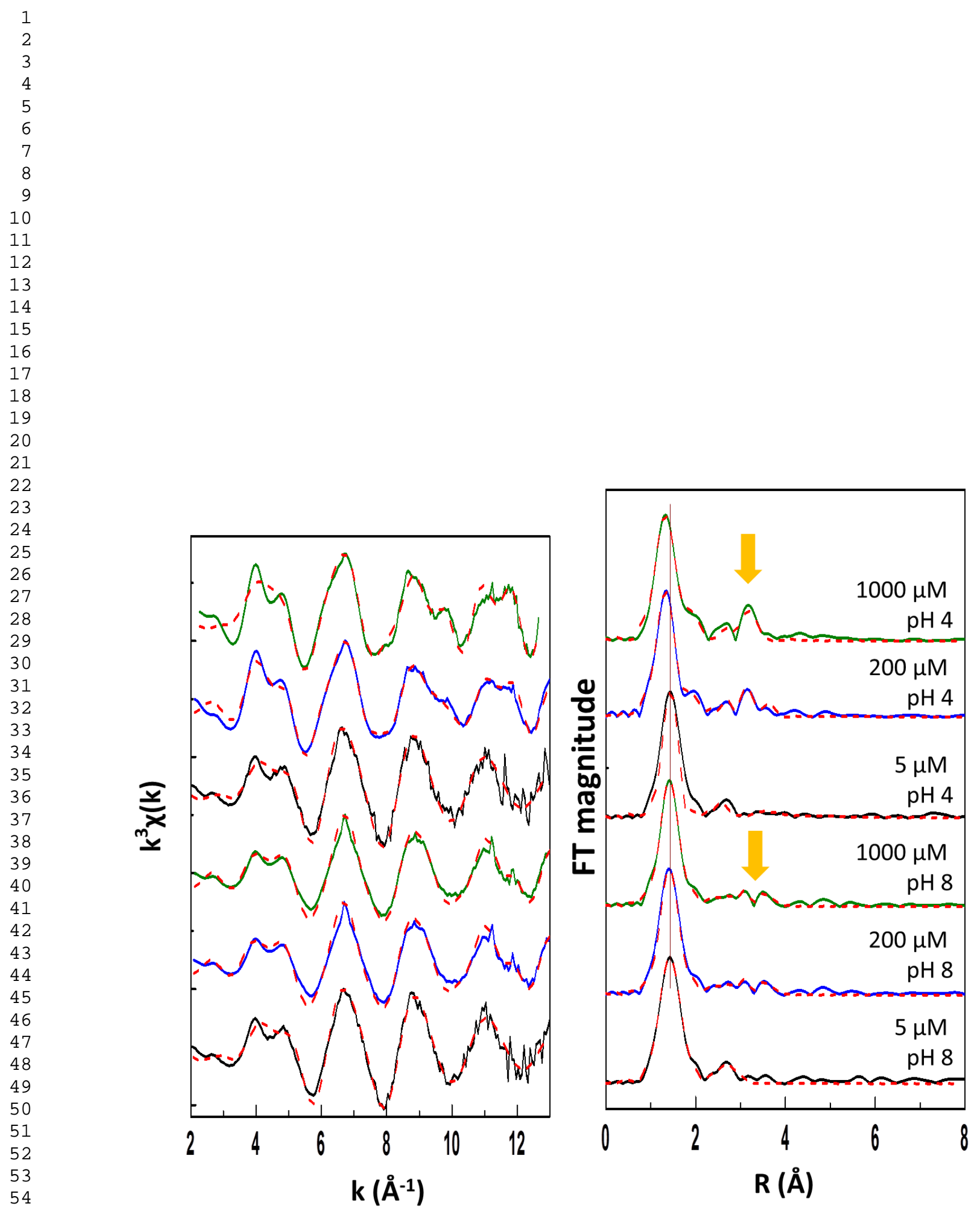

Figure 8. 
a

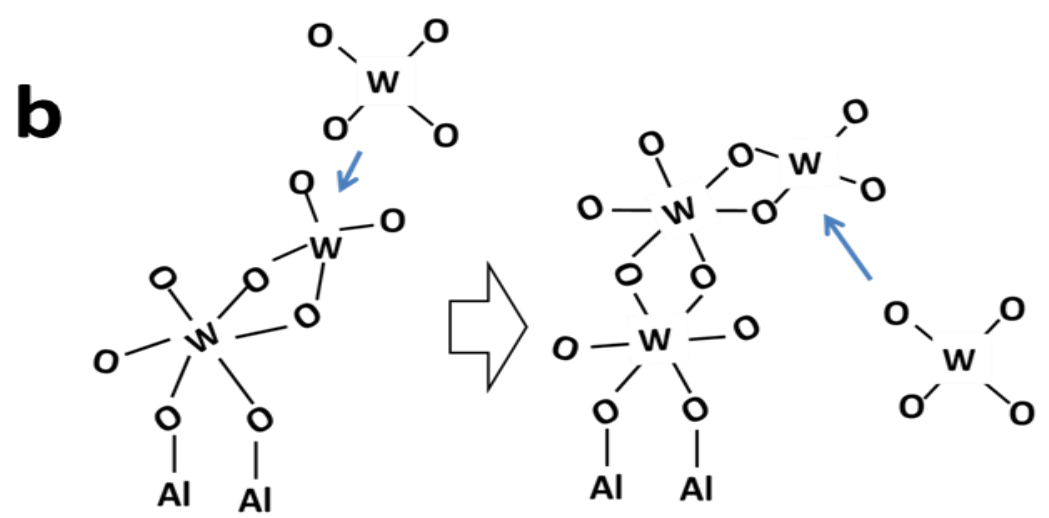

Figure 9. 


\section{List of table captions}

Table 1. Summary of EXAFS fitting results for tungstate-sorbed boehmite samples 
Table 1.Summary of EXAFS fitting results for tungstate-sorbed boehmite samples

\begin{tabular}{|c|c|c|c|c|c|}
\hline Sample & Path & $\mathrm{CN}^{\mathrm{a}}$ & $\mathrm{R}(\AA)^{\mathrm{b}}$ & $\sigma^{2}\left(\AA^{2}\right)^{\mathrm{c}}$ & $\Delta \mathrm{E}_{0}(\mathrm{eV})$ \\
\hline \multirow{3}{*}{$\begin{array}{c}5 \mu \mathrm{M} \\
\mathrm{pH} 4 \text { and } 8\end{array}$} & $\mathrm{~W}-\mathrm{O} 1$ & 3.1 & 1.75 & 0.002 & \multirow{3}{*}{3.4} \\
\hline & $\mathrm{W}-\mathrm{O} 2$ & 3.2 & 2.14 & 0.009 & \\
\hline & $\mathrm{W}-\mathrm{Al}$ & 2.2 & 3.14 & 0.01 & \\
\hline \multirow{5}{*}{$\begin{array}{c}1000 \mu \mathrm{M} \\
\mathrm{pH} 8\end{array}$} & W-O1 & 3.0 & 1.75 & 0.003 & \multirow{5}{*}{-0.4} \\
\hline & $\mathrm{W}-\mathrm{O} 2$ & 4.0 & 2.14 & 0.009 & \\
\hline & $\mathrm{W}-\mathrm{Al}$ & $2 *$ & 3.14 & 0.012 & \\
\hline & W-W1 & 0.6 & 3.23 & 0.004 & \\
\hline & W-W2 & 1.6 & 3.73 & 0.007 & \\
\hline \multirow{6}{*}{$\begin{array}{c}200 \mu \mathrm{M} \\
\mathrm{pH} 4\end{array}$} & $\mathrm{~W}-\mathrm{O} 1$ & $2 *$ & 1.73 & 0.003 & \multirow{6}{*}{-4.1} \\
\hline & $\mathrm{W}-\mathrm{O} 2$ & $2 *$ & 1.90 & 0.019 & \\
\hline & W-O3 & $2 *$ & 2.19 & 0.007 & \\
\hline & $\mathrm{W}-\mathrm{Al}$ & $2 *$ & 3.19 & 0.012 & \\
\hline & W-W & 2.4 & 3.25 & 0.008 & \\
\hline & W-W & 1.3 & 3.69 & 0.008 & \\
\hline \multirow{5}{*}{$\begin{array}{c}1000 \mu \mathrm{M} \\
\mathrm{pH} 4\end{array}$} & $\mathrm{~W}-\mathrm{O} 1$ & $2 *$ & 1.74 & 0.003 & \multirow{5}{*}{-1.2} \\
\hline & $\mathrm{W}-\mathrm{O} 2$ & $2 *$ & 1.98 & 0.019 & \\
\hline & W-O3 & $2 *$ & 2.21 & 0.006 & \\
\hline & $\mathrm{W}-\mathrm{Al}$ & $2 *$ & 3.15 & 0.015 & \\
\hline & W-W & 3.4 & 3.25 & 0.008 & \\
\hline
\end{tabular}

*: fixed in the fitting,

a : coordination number $( \pm 20 \%)$, b: distance in $\AA( \pm 0.02 \AA)$,

c : Debye-Waller factor 